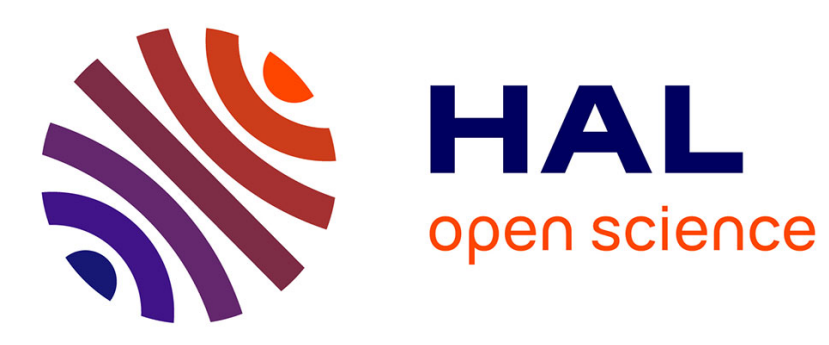

\title{
Dynamic finite element formulation for Cosserat elastic plates
}

Michele Godio, Ioannis Stefanou, Karam Sab, Jean Sulem

\section{To cite this version:}

Michele Godio, Ioannis Stefanou, Karam Sab, Jean Sulem. Dynamic finite element formulation for Cosserat elastic plates. International Journal for Numerical Methods in Engineering, 2015, 101 (13), pp.992-1018. 10.1002/nme.4833 . hal-01114343

\section{HAL Id: hal-01114343 \\ https://hal.science/hal-01114343}

Submitted on 9 Feb 2015

HAL is a multi-disciplinary open access archive for the deposit and dissemination of scientific research documents, whether they are published or not. The documents may come from teaching and research institutions in France or abroad, or from public or private research centers.
L'archive ouverte pluridisciplinaire HAL, est destinée au dépôt et à la diffusion de documents scientifiques de niveau recherche, publiés ou non, émanant des établissements d'enseignement et de recherche français ou étrangers, des laboratoires publics ou privés. 


\title{
Dynamic Finite Element Formulation for Cosserat Elastic Plates
}

\author{
Michele Godio, a *, loannis Stefanou1, b, Karam Sab, c, Jean Sulem ${ }^{1, d}$ \\ ${ }^{1}$ Université Paris-Est, Laboratoire Navier (UMR 8205), CNRS, ENPC, IFSTTAR, F-77455 \\ Marne-la-Vallée, France \\ amichele.godio@enpc.fr, bioannis.stefanou@enpc.fr, ckaram.sab@enpc.fr, djean.sulem@enpc.fr
}

\author{
Keywords: Finite Element methods · Cosserat continuum · Plates · Drilling rotations · Shear \\ locking · Masonry
}

\begin{abstract}
A displacement and rotation-based dynamic Finite Element formulation for Cosserat plates is discussed in detail in this paper. Special attention is given to the validation of the element through adequate benchmarks and patch tests. The choice of the interpolation functions and the order of integration of the stiffness and the mass matrices are extensively argued. The possibility of local system deficiencies is explored and a shear locking investigation specifically elaborated for Cosserat plates is carried out. It is shown how the present formulation has interesting computational properties, as compared to a classical continuum-based formulation, and how it can provide suitable results despite the use of reduced integration. An example of application of the finite element is given, in which the natural frequencies of a masonry panel modelled by means of Discrete Elements are computed and compared with the Finite Elements solution.
\end{abstract}

\section{Introduction}

Cosserat (or micropolar) continuum has recently attracted the attention of researchers from many different disciplines of Mechanics. Either facing it as an adequate mathematical framework for the regularization of ill-posed problems in the softening plasticity regime of classical (Cauchy) continua [1], or as the starting point for the constitutive description of systems with internal lengths, or, again, as a medium with dispersive properties, the theory of Cosserat continuum has found numerous applications up to present.

Models based on Cosserat continuum have been employed, for instance, in the representation of granular media [2]-[4], rock masses [5], [6], block [7]-[9] and layered structures [10], polycrystals and composites materials [10], [11], structures of beams, nanostructures and continuous robotic systems [12]-[14]. Other models have been formulated for the modelling of porous and multiphase materials [15], but also particle fluids and materials with electro- and ferromagnetic properties [16], [17]. Micropolar descriptions are also used in biomechanics, for the mechanical behaviour of bones [18]-[20] and other biological tissues with microstructure [21], [22]. For a more comprehensive review of Cosserat (or micropolar) models for solids and plates, we refer to [23].

Problems involving boundary layer phenomena, localization of deformations and wave dispersion can be properly solved using Cosserat Finite Elements (FE). Sluys and de Borst presented a comprehensive study on the advantages of Cosserat FE compared to classical formulations based on Cauchy continuum, which suffer from the problem of mesh dependency when localization of shear strains takes place, see [24]-[26] among others. Interesting applications of Cosserat FE in modelling layered structures are given by [27]-[29]. Cosserat FE for masonry structures are also formulated in [30]-[33]. In modeling masonry, it is the non-symmetry of the stress tensor that makes Cosseratcontinuum-based models preferable [34] with respect to models based on other non-classical continua. Other more general FE formulations for micropolar plates and shells and for solids with microstructure are those recently proposed by [35], [36] and [37]-[40].

However, even though many applications of Cosserat continuum using FE are found in the literature, the details of each FE formulation are partially exposed or not presented at all. Moreover, the performance of the element is seldom investigated, making hard to assess the chosen FE formulation and its applicability to other problems. The purpose of this paper is to propose a Cosserat finite element that may be used for different applications in structural and materials engineering.

The presented element is obtained by the superposition of a quadratic small-strains Cosserat membrane rectangle with a thick plate element with enriched kinematics. It results in a versatile shell-type flat element equipped with six degrees of freedom (DOF) per node, capable of modelling complex spatial structural configurations.

This Cosserat finite element has the advantage of providing accurate results even in the case of reduced integration, without using any hourglass control method. In classic FE formulations the hourglass control is frequently made by introducing an artificial stiffness to the in-plane rotations [41]-[46]. In FE formulations based on Cosserat continuum, this stiffness is introduced in a physical way, because of the inherent drilling rotational DOF. The occurrence of zeroenergy modes can be thus naturally avoided in the Cosserat FE formulations, and the element stiffness matrix is never deficient. 
The paper has the following structure. In Section 2 we outline the basic definitions and relations of a Cosserat continuum in three dimensions that are necessary for the rest of the paper. In Section 3 we present a model for micropolar plates, through reduction of the three-dimensional problem. In Section 4 we propose a general Cosserat dynamic formulation for the FE method. In Section 5 we present a specific finite element: the choice of the interpolation order is discussed, the use of different integration techniques for the computation of the element stiffness and mass matrices are argued and the possibility of system deficiencies is explored. The validation of the element is made in Section 6, where we investigate the shear locking phenomenon applied to micropolar plates. In Section 7 we give an illustrative example of the use of the element in representing the modal response of a masonry structure consisted of discrete interacting building blocks.

Throughout the paper much attention is paid to the capacity of the formulation to predict the membrane (or inplane) as well as the flexural (or out-of-plane) behaviour. Even though from a theoretical point of view they will be presented separately, only their superposition give the full, herein referred to as plate, structural response.

Common matrix notation is adopted. Upper case letters denote variables referring to the nodes of the element, while lower case letters refer to the local variables of the continuum field problem. It is implicitly assumed that the former are only time-dependent whereas the latter are space and time-dependent. Partial differentiation with respect to orthogonal coordinates is indicated by $[\cdot]_{i, j}=\partial[\cdot]_{i} / \partial x_{j}$. Time derivative is $\left[{ }^{\vdots}\right]_{i}=[\cdot]_{i, t}$.

\section{3D Cosserat dynamics prerequisites}

Following Germain's terminology [47], Cosserat continuum is a special case of a micromorphic continuum of first order (Fig. 1). Therein, the particle (the material point in the sense of Germain) is considered rigid and, consequently, its kinematical description in the three-dimensional (3D) space is fulfilled by six DOF, i.e. three translations $u_{i}$ and three rotations $\omega_{i}$.

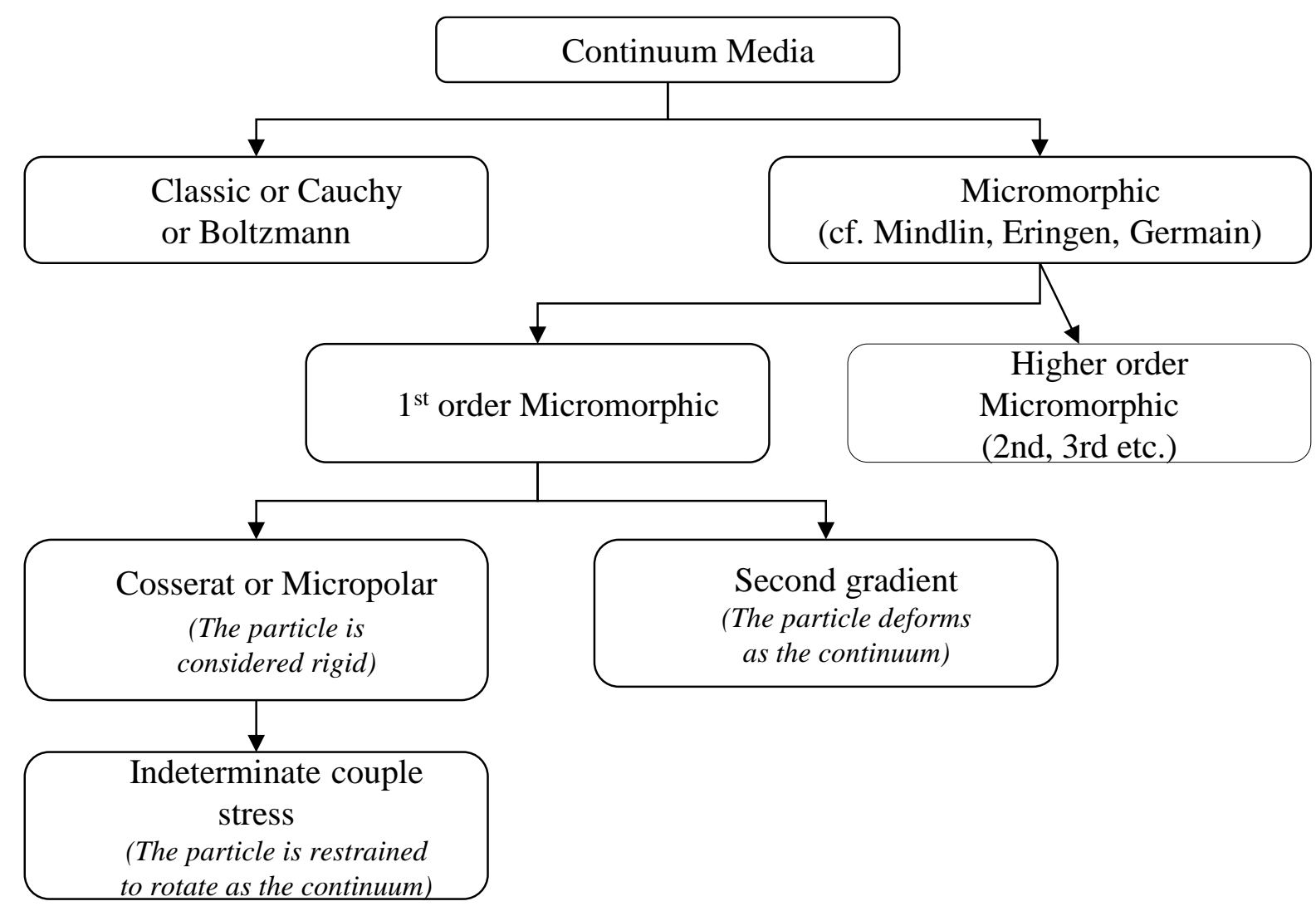

Fig. 1 Higher order continuum theories according to Germain [47] terminology. Cosserat continuum is a special case of a micromorphic continuum of first order.

It results that the deformation measures of the medium, herein expressed in Cartesian coordinates and in the frame of a small strain theory, are given by two second order tensors, $\gamma_{i j}$ and $\kappa_{i j}$, representing respectively the relative strains and the curvatures: 


$$
\begin{aligned}
& \gamma_{i j}=u_{i, j}+e_{i j k} \omega_{k} \\
& \kappa_{i j}=\omega_{i, j},
\end{aligned}
$$

with $e_{i j k}$ the permutation symbol. The symmetric part of the strain tensor, denoted with $\gamma_{(i j)}$, coincides with the strain tensor of a classical (Cauchy) continuum, whereas its skew-symmetric part $\gamma_{[i j]}$ accounts for the relative deformations:

$$
\begin{aligned}
& \gamma_{(i j)}=\frac{\left(u_{i, j}+u_{j, i}\right)}{2} \\
& \gamma_{[i j]}=e_{i j k}\left(\widehat{\omega}_{k}-\omega_{k}\right),
\end{aligned}
$$

where $e_{i j k} \widehat{\omega}_{k}=\frac{u_{j, i}-u_{i, j}}{2}$ is the infinitesimal rotation tensor.

The above deformation measures are energy conjugate with the non-symmetric stresses $\tau_{i j}$ and the couple stresses $\mu_{i j}$. According to Boltzmann, the assumption of symmetry of the stress tensor in classical continua has an axiomatic character (see [48], [49]). In the Cosserat medium, the loss of symmetry is due to the rotational DOF attached to the particle, as shown by Eq.(4), and the presence of the couple stresses (angular momentum). This makes rich the number of the material parameters to be identified within the constitutive relations, and makes Cosserat continuum suitable for the description of a great variety of problems. For instance, for a centrosymmetric material, a constitutive law can be defined in a general manner as:

$$
\begin{aligned}
\tau_{i j} & =C_{i j k l} \gamma_{k l} \\
\mu_{i j} & =D_{i j k l} \kappa_{k l}
\end{aligned}
$$

where $C_{i j k l}$ and $D_{i j k l}$ are fourth order tensors containing the material parameters. In this case, an isotropic micropolar material require the definition of six parameters, whereas a classical solid would only demand two moduli [23].

For a Cosserat continuum, the Cauchy tetrahedron can be generalized as follows [47]:

$$
\begin{gathered}
T_{i}^{d}=\tau_{i j} n_{j} \\
M_{i}^{d}=\mu_{i j} n_{j},
\end{gathered}
$$

where $T_{i}^{d}$ and $M_{i}^{d}$ are the stress and the couple stress vectors applied on the boundary $\partial \Omega_{\Sigma}$ of a configuration $\Omega$, and $n_{j}$ is the normal outward unit vector at $\partial \Omega_{\Sigma}$. Moreover, both translations and rotations can be prescribed on the boundary $\partial \Omega_{U}$ :

$$
\begin{aligned}
& u_{i}=u_{i}^{d} \\
& \omega_{i}=\omega_{i}^{d} .
\end{aligned}
$$

In Statics, the minimum of the Total Potential Energy gives the equilibrium equations in weak form. In Dynamics, the equations of motion can be derived by application of the Hamilton's variational Principle. For a virtual variation of the displacement $\delta u_{i}$ and the rotational field $\delta \omega_{i}$, the Principle reads, in case of a conservative holonomic continuum systems:

$$
\delta \int_{t_{1}}^{t_{2}}\left[\mathrm{~K}^{c}-\left(\mathrm{V}^{c}-\mathrm{W}^{c}\right)\right] \mathrm{d} t=0 .
$$

In Eq.(8), we recognise the kinetic energy $\mathrm{K}^{c}$, the deformation energy $\mathrm{V}^{c}$, and the expression for the external works $\mathrm{W}^{c}$. In terms of Cosserat kinematics, these quantities read:

$$
\begin{aligned}
& \mathrm{V}^{c}=\frac{1}{2} \int_{\Omega}\left(\tau_{i j} \gamma_{i j}+\mu_{i j} \kappa_{i j}\right) \mathrm{d} \Omega \\
& \mathrm{W}^{c}=\int_{\Omega}\left(f_{i} u_{i}+m_{i} \omega_{i}\right) \mathrm{d} \Omega+\int_{\partial \Omega_{\Sigma}}\left(T_{i}^{d} u_{i}+M_{i}^{d} \omega_{i}\right) \mathrm{d} A \\
& \mathrm{~K}^{c}=\frac{1}{2} \int_{\Omega}\left(\rho \dot{u}_{i} \dot{u}_{i}+\rho \dot{\omega}_{i} I_{i j} \dot{\omega}_{j}\right) \mathrm{d} \Omega .
\end{aligned}
$$

In the above, the body forces $f_{i}$ and the body couples $m_{i}$ represent long-range actions within the continuum. The material mass density is $\rho$, and the micro-inertia tensor is $\rho I_{i j}$. The linear and angular momentum balance is then: 


$$
\begin{aligned}
& \tau_{i j, j}+f_{i}-\rho \ddot{u}_{i}=0 \\
& \mu_{i j, j}-e_{i j k} \tau_{j k}+m_{i}-\rho I_{i j} \ddot{\omega}_{j}=0 .
\end{aligned}
$$

\section{Equations of motion for Cosserat elastic plates}

The 3D Cosserat field's problem presented in Section 2 is governed by six partial differential equations (PDE) of second order, expressed in terms of the three unknown displacements and the three unknown rotations, see Eqs.(12). In the case of a plate, a homogeneous planar surface of unitary thickness $h=1$ oriented in the 1-2 plane, the problem is invariant in the out-of-plane direction $x_{3}$ and so it can be consequently reduced (Fig. 2). Regarding the curvatures, we have $\kappa_{13}=\kappa_{23}=\kappa_{33}=0$, which annihilate the conjugate couple stress measures $\mu_{13}, \mu_{23}$ and $\mu_{33}$. Concerning the relative strains, $\gamma_{33}$ vanishes due to the aforementioned hypothesis, and components $\gamma_{13}$ and $\gamma_{23}$ result in the simplified expressions:

$$
\begin{aligned}
& \gamma_{13}=-\omega_{2} \\
& \gamma_{23}=\omega_{1} .
\end{aligned}
$$

A typical assumption which is often taken in the construction of classical and micropolar plate theories and that we also adopt for our formulation, consists in neglecting the normal stress $\tau_{33}$ (see [50] for a further discussion). However Eq.(13) preserves the stress components $\tau_{13}$ and $\tau_{23}$. Although such components are absent in most of the models designed for Cosserat plates, see for instance those obtained by reduction of the 3D problem [51]-[53], they are conserved in the present formulation. This allows for the implementation of more complete plate models, as the one proposed by Eringen [50].

Dealing with plates, it is handy to separate the membrane (or in-plane) behaviour from the flexural (or out-ofplane) behaviour, see Fig. 2. The static and kinematic remaining components are expressed in matrix notation in Table 1 , where subscripts $m$ and $f$ refer respectively to the variables associated with the membrane and flexural behaviour. Regarding the membrane behaviour, we recognise the tractions $\tau_{\alpha \beta}(\alpha=\beta$, with $\alpha, \beta=1,2)$, the in-plane shears $\tau_{\alpha \beta}$ $(\alpha \neq \beta)$, and the in-plane couples $\mu_{3 \alpha}$. Concerning the flexural behaviour, we identify the torsions $\mu_{\alpha \beta}(\alpha=\beta)$, the out-of-plane flexions $\mu_{\alpha \beta}(\alpha \neq \beta)$, the transverse shears $\tau_{3 \alpha}$, and the longitudinal shears $\tau_{\alpha 3}$.
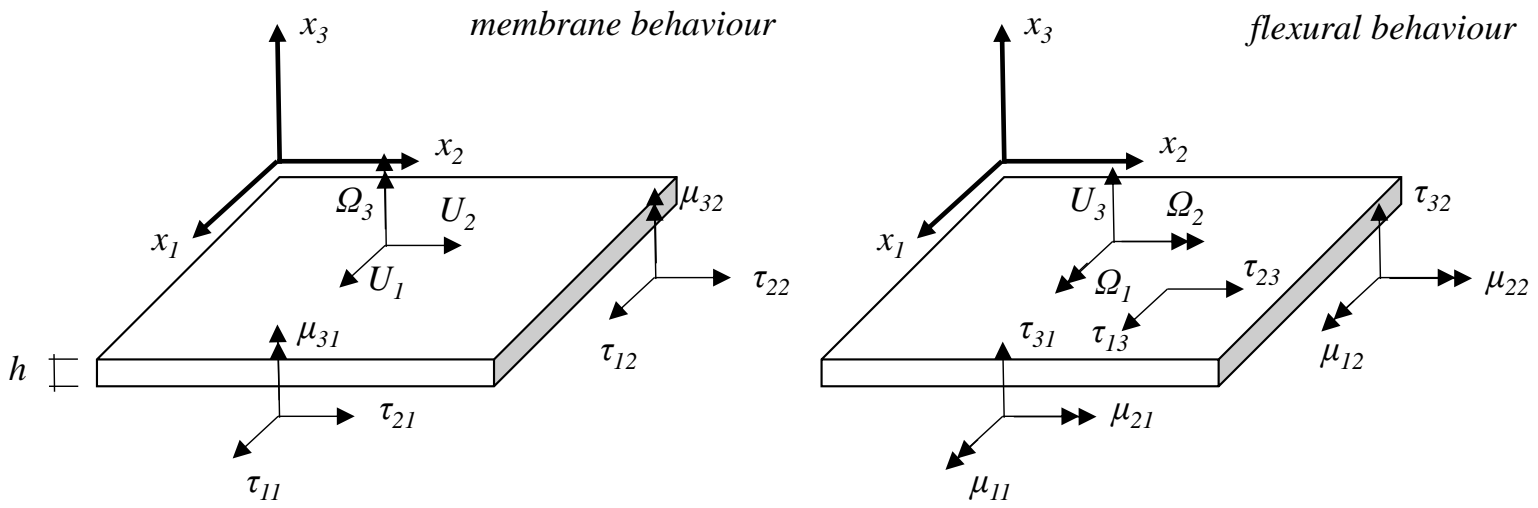

Fig. 2 Stresses, couple stresses and degrees of freedom of a Cosserat plate: membrane (left) and flexural (right) behaviour.

Following this notation, the PDE for the in- and out-of-plane motion of Cosserat plates read respectively, in compact form:

$$
\left[\begin{array}{rr}
\boldsymbol{L}_{\boldsymbol{m} \mathbf{1}[2 \times 4]^{t}}{ }^{t} & -\boldsymbol{L}_{\boldsymbol{m} 3[2 \times 2]}{ }^{t} \\
-\boldsymbol{L}_{\boldsymbol{m}[1 \times 4]} & \boldsymbol{L}_{\boldsymbol{m} 4[1 \times 2]}
\end{array}\right]\left[\begin{array}{c}
\boldsymbol{\tau}_{\boldsymbol{m}} \\
\boldsymbol{\mu}_{\boldsymbol{m}}
\end{array}\right]+\left[\begin{array}{c}
\boldsymbol{f}_{\boldsymbol{m}} \\
m_{m}
\end{array}\right]-\left[\begin{array}{rr}
\mathbf{1}_{\boldsymbol{m}[2 \times 2]} & \mathbf{0}_{[2 \times 1]} \\
\mathbf{0}_{[1 \times 2]} & I_{m[1 \times 1]}
\end{array}\right]\left[\begin{array}{c}
\ddot{\boldsymbol{u}}_{\boldsymbol{m}} \\
\ddot{\boldsymbol{\omega}}_{m}
\end{array}\right]=\mathbf{0}
$$

and

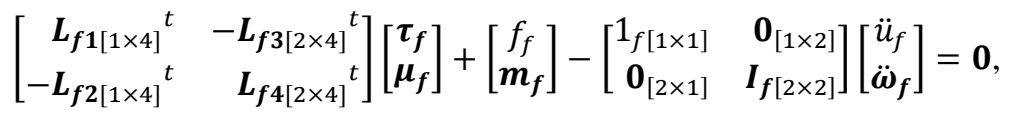

where the sub-matrices $\boldsymbol{L}_{\boldsymbol{m} i}$ and $\boldsymbol{L}_{\boldsymbol{f} i}$ are the operators giving the definition of the deformation measures of the plate (Eq.(1)-(2)): 


$$
\begin{aligned}
& {\left[\begin{array}{l}
\gamma_{m} \\
\boldsymbol{\kappa}_{\boldsymbol{m}}
\end{array}\right]=\left[\begin{array}{ll}
\boldsymbol{L}_{\boldsymbol{m} 1[4 \times 2]} & \boldsymbol{L}_{\boldsymbol{m} 2[4 \times 1]} \\
\boldsymbol{L}_{\boldsymbol{m} 3[2 \times 2]} & \boldsymbol{L}_{\boldsymbol{m}[2 \times 1]}
\end{array}\right]\left[\begin{array}{l}
\boldsymbol{u}_{\boldsymbol{m}} \\
\omega_{m}
\end{array}\right]} \\
& {\left[\begin{array}{l}
\boldsymbol{\gamma}_{f} \\
\boldsymbol{\kappa}_{f}
\end{array}\right]=\left[\begin{array}{ll}
\boldsymbol{L}_{\boldsymbol{f} 1[4 \times 1]} & \boldsymbol{L}_{\boldsymbol{f} 2[4 \times 2]} \\
\boldsymbol{L}_{\boldsymbol{f} 3[4 \times 1]} & \boldsymbol{L}_{\boldsymbol{f}[4 \times 2]}
\end{array}\right]\left[\begin{array}{l}
u_{f} \\
\boldsymbol{\omega}_{f}
\end{array}\right],}
\end{aligned}
$$

that is:

$$
\begin{aligned}
& \boldsymbol{L}_{\boldsymbol{m} \mathbf{1}[4 \times 2]}=\left[\begin{array}{cc}
{[\cdot]_{, 1}} & 0 \\
0 & {[\cdot]_{, 2}} \\
{[\cdot]_{, 2}} & 0 \\
0 & {[\cdot]_{, 1}}
\end{array}\right] \quad \boldsymbol{L}_{\boldsymbol{m} 2[4 \times 1]}=\left[\begin{array}{r}
0 \\
0 \\
+1 \\
-1
\end{array}\right] \quad \boldsymbol{L}_{\boldsymbol{m} 3[2 \times 2]}=\left[\begin{array}{ll}
0 & 0 \\
0 & 0
\end{array}\right] \quad \boldsymbol{L}_{\boldsymbol{m} 4[2 \times 1]}=\left[\begin{array}{l}
{[\cdot]_{, 1}} \\
{[\cdot]_{, 2}}
\end{array}\right], \\
& \boldsymbol{L}_{\boldsymbol{f} 1[4 \times 1]}=\left[\begin{array}{c}
0 \\
{[\cdot]_{, 1}} \\
0 \\
{[\cdot]_{, 2}}
\end{array}\right] \quad \boldsymbol{L}_{\boldsymbol{f} 2[4 \times 2]}=\left[\begin{array}{rr}
0 & -1 \\
0 & +1 \\
+1 & 0 \\
-1 & 0
\end{array}\right] \quad \boldsymbol{L}_{\boldsymbol{f} 3[4 \times 1]}=\left[\begin{array}{l}
0 \\
0 \\
0 \\
0
\end{array}\right] \quad \boldsymbol{L}_{\boldsymbol{f} 4[4 \times 2]}=\left[\begin{array}{cc}
{[\cdot]_{, 1}} & 0 \\
0 & {[\cdot]_{, 2}} \\
{[\cdot]_{, 2}} & 0 \\
0 & {[\cdot]_{, 1}}
\end{array}\right] \text {. }
\end{aligned}
$$

In a plate, material symmetries can be defined only with respect to the normal axis $x_{3}$ [54], [55]. Therefore, without losing generality a constitutive law is introduced in the following form:

$$
\begin{aligned}
& {\left[\begin{array}{l}
\boldsymbol{\tau}_{\boldsymbol{m}} \\
\boldsymbol{\mu}_{\boldsymbol{m}}
\end{array}\right]=\left[\begin{array}{ll}
\boldsymbol{A}_{[4 \times 4]} & \boldsymbol{G}_{[4 \times 2]} \\
\boldsymbol{H}_{[2 \times 4]} & \boldsymbol{D}_{\boldsymbol{m}[2 \times 2]}
\end{array}\right]\left[\begin{array}{l}
\boldsymbol{\gamma}_{\boldsymbol{m}} \\
\boldsymbol{\kappa}_{\boldsymbol{m}}
\end{array}\right]} \\
& {\left[\begin{array}{l}
\boldsymbol{\tau}_{\boldsymbol{f}} \\
\boldsymbol{\mu}_{\boldsymbol{f}}
\end{array}\right]=\left[\begin{array}{ll}
\boldsymbol{F}_{[4 \times 4]} & \boldsymbol{0}_{[4 \times 4]} \\
\boldsymbol{0}_{[4 \times 4]} & \boldsymbol{D}_{f[4 \times 4]}
\end{array}\right]\left[\begin{array}{l}
\boldsymbol{\gamma}_{\boldsymbol{f}} \\
\boldsymbol{\kappa}_{\boldsymbol{f}}
\end{array}\right] .}
\end{aligned}
$$

In the above: $\boldsymbol{A}$ governs the membrane behaviour; $\boldsymbol{D}_{\boldsymbol{f}}$ and $\boldsymbol{D}_{\boldsymbol{m}}$ control the in- and out-of-plane bending response; $\boldsymbol{F}$ accounts for the transversal and the longitudinal shears. Matrices $\boldsymbol{G}$ and $\boldsymbol{H}$ are responsible for the coupling between the stress and couple stress components. Next, we will focus on the case of Cosserat materials possessing centrosymmetric properties. In such case, those matrices are considered zero. For a more complete FE formulation, the reader is referred to Appendix 1.

\section{Element formulation}

In Cosserat FE, nodes are equipped with translational and rotational DOF. The number of nodes to which refer the translations and the rotations depends to the degree of interpolation attended for those fields. As it will be discussed in Section 5.1, many choices are possible in this sense. In order to keep the formulation general, we assume that the element translations are defined on $\mathrm{N}$ nodes, whereas element rotations are attached to $\mathrm{M}$ nodes. Therefore, the vectors containing the nodal variables of the element can be written as:

$$
\begin{aligned}
& {\left[\begin{array}{ll}
\boldsymbol{U}_{\boldsymbol{m}}{ }^{t} & \boldsymbol{\Omega}_{\boldsymbol{m}}{ }^{t}
\end{array}\right]=\left[\begin{array}{llllll}
\boldsymbol{U}_{\boldsymbol{m}}^{\mathbf{1}^{t}} & \ldots & \boldsymbol{U}_{\boldsymbol{m}}^{\mathbf{N}^{t}} & \Omega_{m}^{1} & \ldots & \Omega_{m}^{\mathrm{M}}
\end{array}\right]} \\
& {\left[\begin{array}{llllll}
\boldsymbol{U}_{\boldsymbol{f}}{ }^{t} & \boldsymbol{\Omega}_{\boldsymbol{f}}{ }^{t}
\end{array}\right]=\left[\begin{array}{llllll}
U_{f}{ }^{1} & \ldots & U_{f}^{\mathrm{N}} & \boldsymbol{\Omega}_{\boldsymbol{f}}^{\mathbf{1}^{t}} & \ldots & \boldsymbol{\Omega}_{\boldsymbol{f}}^{\mathbf{M}^{t}}
\end{array}\right]}
\end{aligned}
$$

The aforementioned $\mathrm{N}$ nodal displacements and the $\mathrm{M}$ nodal rotations are interpolated by means of general shape functions $\boldsymbol{N}$ and $\boldsymbol{\Phi}$, respectively:

$$
\begin{aligned}
& {\left[\begin{array}{l}
\boldsymbol{u}_{\boldsymbol{m}} \\
\omega_{m}
\end{array}\right]=\left[\begin{array}{rr}
\boldsymbol{N}_{\boldsymbol{m}[2 \times 2 N]} & \boldsymbol{0}_{[2 \times M]} \\
\mathbf{0}_{[1 \times 2 N]} & \boldsymbol{\Phi}_{\boldsymbol{m}[1 \times M]}
\end{array}\right]\left[\begin{array}{l}
\boldsymbol{U}_{\boldsymbol{m}} \\
\boldsymbol{\Omega}_{\boldsymbol{m}}
\end{array}\right]} \\
& {\left[\begin{array}{l}
u_{f} \\
\boldsymbol{\omega}_{\boldsymbol{f}}
\end{array}\right]=\left[\begin{array}{rr}
\boldsymbol{N}_{\boldsymbol{f}[1 \times N]} & \mathbf{0}_{[1 \times 2 M]} \\
\mathbf{0}_{[2 \times N]} & \boldsymbol{\Phi}_{f[2 \times 2 M]}
\end{array}\right]\left[\begin{array}{l}
\boldsymbol{U}_{\boldsymbol{f}} \\
\boldsymbol{\Omega}_{\boldsymbol{f}}
\end{array}\right] .}
\end{aligned}
$$

In particular, $\boldsymbol{N}_{\boldsymbol{m}}$ interpolate the in-plane displacements, $\boldsymbol{N}_{\boldsymbol{f}}$ the out-of-plane displacements, $\boldsymbol{\Phi}_{\boldsymbol{m}}$ the in-plane rotational field and $\boldsymbol{\Phi}_{f}$ the out-of-plane rotational fields: 


$$
\begin{aligned}
& \boldsymbol{N}_{\boldsymbol{m}[2 \times 2 N]}=\left[\begin{array}{ccccc}
N_{1} & 0 & & N_{N} & 0 \\
0 & N_{1} & \ldots & 0 & N_{N}
\end{array}\right] \quad \boldsymbol{N}_{f[1 \times N]}=\left[\begin{array}{lll}
N_{1} & \ldots & N_{N}
\end{array}\right] \\
& \boldsymbol{\Phi}_{\boldsymbol{m}[1 \times M]}=\left[\begin{array}{lll}
\Phi_{1} & \ldots & \Phi_{M}
\end{array}\right] \quad \boldsymbol{\Phi}_{f[2 \times 2 M]}=\left[\begin{array}{ccccc}
\Phi_{1} & 0 & & \Phi_{M} & 0 \\
0 & \Phi_{1} & \ldots & 0 & \Phi_{M}
\end{array}\right] .
\end{aligned}
$$

It follows that the expressions of the deformation measures within the FE are:

$$
\begin{aligned}
& {\left[\begin{array}{l}
\boldsymbol{\gamma}_{\boldsymbol{m}} \\
\boldsymbol{\kappa}_{\boldsymbol{m}}
\end{array}\right]=\left[\begin{array}{ll}
\boldsymbol{B}_{\boldsymbol{m} 1[4 \times 2 N]} & \boldsymbol{B}_{\boldsymbol{m} 2[4 \times M]} \\
\boldsymbol{B}_{\boldsymbol{m}[2 \times 2 N]} & \boldsymbol{B}_{\boldsymbol{m} 4[2 \times M]}
\end{array}\right]\left[\begin{array}{l}
\boldsymbol{U}_{\boldsymbol{m}} \\
\boldsymbol{\Omega}_{\boldsymbol{m}}
\end{array}\right]} \\
& {\left[\begin{array}{l}
\boldsymbol{\gamma}_{\boldsymbol{f}} \\
\boldsymbol{\kappa}_{\boldsymbol{f}}
\end{array}\right]=\left[\begin{array}{ll}
\boldsymbol{B}_{\boldsymbol{f}[4 \times N]} & \boldsymbol{B}_{\boldsymbol{f}[44 \times 2 M]} \\
\boldsymbol{B}_{f 3[4 \times N]} & \boldsymbol{B}_{\boldsymbol{f} 4[4 \times 2 M]}
\end{array}\right]\left[\begin{array}{l}
\boldsymbol{U}_{\boldsymbol{f}} \\
\boldsymbol{\Omega}_{\boldsymbol{f}}
\end{array}\right],}
\end{aligned}
$$

where the definition of compliant matrices $\boldsymbol{B}_{\boldsymbol{\alpha} \mathbf{i}}$ can be found by introducing Eq.(24) into Eq.(16)-(17), and obtaining, in very compact form, the following relations:

$$
\begin{array}{ll}
\boldsymbol{B}_{\alpha \mathrm{i}}=\boldsymbol{L}_{\boldsymbol{\alpha}} \boldsymbol{N}_{\boldsymbol{\alpha}}, & \text { for } i=1,3 \\
\boldsymbol{B}_{\boldsymbol{\alpha} \mathrm{i}}=\boldsymbol{L}_{\boldsymbol{\alpha} \mathbf{i}} \boldsymbol{\Phi}_{\alpha}, & \text { for } i=2,4
\end{array} \quad \text { with } \alpha=m, f
$$

Notice that matrices $\boldsymbol{B}_{\boldsymbol{m} 2}$ and $\boldsymbol{B}_{\boldsymbol{m} 4}$ are associated with the in-plane (or drilling) nodal rotations $\Omega_{3}$, which are absent in the classical engineering plate theories.

\subsection{Stiffness matrix}

The substitution of the relations (22)-(23) and (25)-(26) into the expressions of the deformation energy $\mathrm{V}^{c}$ (from Eq.(9)) and of the external works $\mathrm{W}^{c}$ (from Eq.(10)) for the plate, leads to the definitions of the element stiffness matrix and the nodal load vectors. Adopting the notation used by [56] for the in-plane behaviour, it holds:

$$
\mathrm{V}_{m}^{c}=\frac{1}{2}\left[\begin{array}{ll}
\boldsymbol{U}_{\boldsymbol{m}}{ }^{t} & \boldsymbol{\Omega}_{\boldsymbol{m}}{ }^{t}
\end{array}\right]\left[\begin{array}{ll}
\boldsymbol{K}_{U U}^{\boldsymbol{m}} & \boldsymbol{K}_{U \Omega}^{m} \\
\boldsymbol{K}_{\boldsymbol{\Omega} \boldsymbol{U}}^{\boldsymbol{m}} & \boldsymbol{K}_{\boldsymbol{\Omega} \Omega}^{m}
\end{array}\right]\left[\begin{array}{l}
\boldsymbol{U}_{\boldsymbol{m}} \\
\boldsymbol{\Omega}_{\boldsymbol{m}}
\end{array}\right] \quad \mathrm{W}_{m}^{c}=\left[\begin{array}{ll}
\boldsymbol{P}_{\boldsymbol{m}}{ }^{t} & \boldsymbol{Q}_{\boldsymbol{m}}{ }^{t}
\end{array}\right]\left[\begin{array}{l}
\boldsymbol{U}_{\boldsymbol{m}} \\
\boldsymbol{\Omega}_{\boldsymbol{m}}
\end{array}\right]
$$

with the stiffness sub-matrices:

$$
\begin{aligned}
& \boldsymbol{K}_{U \boldsymbol{U}}^{\boldsymbol{m}}=\int_{\Omega^{e}}\left[\boldsymbol{B}_{\boldsymbol{m 1}[2 N \times 4]}^{t} \boldsymbol{A}_{[4 \times 4]} \boldsymbol{B}_{m 1[4 \times 2 N]}\right] \mathrm{d} A
\end{aligned}
$$

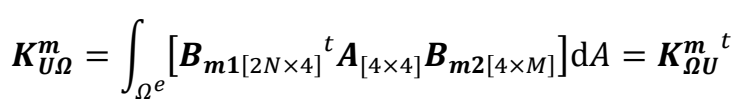

$$
\begin{aligned}
& \boldsymbol{K}_{\Omega \Omega}^{\boldsymbol{m}}=\int_{\Omega^{e}}\left[\boldsymbol{B}_{\boldsymbol{m} 2[M \times 4]} \boldsymbol{A}_{[4 \times 4]} \boldsymbol{B}_{\boldsymbol{m} 2[4 \times M]}+\boldsymbol{B}_{\boldsymbol{m} 4[M \times 2]}^{t} \boldsymbol{D}_{\boldsymbol{m}[2 \times 2]} \boldsymbol{B}_{\boldsymbol{m} 4[2 \times M]}\right] \mathrm{d} A,
\end{aligned}
$$

and the nodal load vectors:

$$
\left[\begin{array}{l}
\boldsymbol{P}_{\boldsymbol{m}} \\
\boldsymbol{Q}_{\boldsymbol{m}}
\end{array}\right]=\int_{\Omega^{e}}\left[\begin{array}{rr}
\boldsymbol{N}_{\boldsymbol{m}[2 N \times 2]^{t}} & \mathbf{0}_{[2 N \times 1]} \\
\mathbf{0}_{[M \times 2]} & \boldsymbol{\Phi}_{\boldsymbol{m}[M \times 1]}^{t}
\end{array}\right]\left[\begin{array}{c}
\boldsymbol{f}_{\boldsymbol{m}} \\
m_{m}
\end{array}\right] \mathrm{d} A+\int_{\partial \Omega_{\Sigma}^{e}}\left[\begin{array}{rr}
\boldsymbol{N}_{\boldsymbol{m}[2 N \times 2]}^{t} & \mathbf{0}_{[2 N \times 1]} \\
\mathbf{0}_{[M \times 2]} & \boldsymbol{\Phi}_{\boldsymbol{m}[M \times 1]}^{t}
\end{array}\right]\left[\begin{array}{c}
\boldsymbol{T}_{\boldsymbol{m}}^{\boldsymbol{d}} \\
M_{m}^{d}
\end{array}\right] \mathrm{d} S .
$$

defined on the element configuration $\Omega^{e}$. The vectors $\boldsymbol{T}_{m}^{d}, M_{m}^{d}$ represent the in-plane forces and moments prescribed on the boundary $\partial \Omega_{\Sigma}^{e}$ of the finite element. It is worth mentioning that the sub-matrix $\boldsymbol{K}_{U U}^{m}$ represents the stiffness matrix of a classical membrane element, whereas $\boldsymbol{K}_{U \Omega}^{m}$ and $\boldsymbol{K}_{\Omega \Omega}^{m}$ are additional terms associated to the drilling rotations. This is the difference between the present Cosserat FE formulation and a classical Cauchy-continuum-based formulation, as far it concerns the in-plane behaviour. As it will be shown in Section 5.2, the first term of $\boldsymbol{K}_{\Omega \Omega}^{m}$ alleviates the local stiffness matrix rank deficiency that occurs in case of reduced integration of classical elements against membrane actions.

The out-of-plane behaviour can be separated into pure bending $(b)$ and shear $(s)$ components. In this case the stiffness matrix is:

$$
K_{f}=\left[\begin{array}{ll}
K_{U U}^{s} & K_{U \Omega}^{s} \\
K_{\Omega U}^{s} & K_{\Omega \Omega}^{s}+K_{\Omega \Omega}^{b}
\end{array}\right],
$$

with 


$$
\begin{aligned}
& \boldsymbol{K}_{\boldsymbol{U U}}^{\boldsymbol{S}}=\int_{\Omega} e\left[\boldsymbol{B}_{\boldsymbol{f} \mathbf{1}[N \times 4]}{ }^{t} \boldsymbol{F}_{[4 \times 4]} \boldsymbol{B}_{\boldsymbol{f} 1[4 \times N]}\right] \mathrm{d} A \\
& \boldsymbol{K}_{U \Omega}^{\boldsymbol{s}}=\int_{\Omega} e\left[\boldsymbol{B}_{f \mathbf{1}[N \times 4]}{ }^{t} \boldsymbol{F}_{[4 \times 4]} \boldsymbol{B}_{f 2[4 \times 2 M]}\right] \mathrm{d} A=\boldsymbol{K}_{\Omega \boldsymbol{U}}^{\boldsymbol{s}}{ }^{t}
\end{aligned}
$$

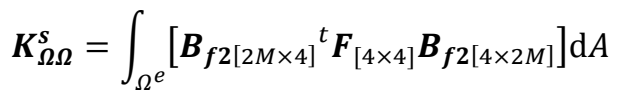

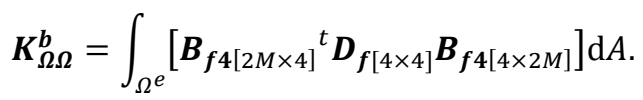

The nodal load vectors are:

$$
\left[\begin{array}{l}
\boldsymbol{P}_{\boldsymbol{f}} \\
\boldsymbol{Q}_{\boldsymbol{f}}
\end{array}\right]=\int_{\Omega^{e}}\left[\begin{array}{cr}
\boldsymbol{N}_{\boldsymbol{f}[N \times 1]}^{t} & \mathbf{0}_{[N \times 2]} \\
\mathbf{0}_{[2 M \times 1]} & \boldsymbol{\Phi}_{\boldsymbol{f}[2 M \times 2]}^{t}
\end{array}\right]\left[\begin{array}{c}
f_{f} \\
\boldsymbol{m}_{\boldsymbol{f}}
\end{array}\right] \mathrm{d} A+\int_{\partial \Omega_{\Sigma}^{e}}\left[\begin{array}{lr}
\boldsymbol{N}_{\boldsymbol{f}[N \times 1]}^{t} & \mathbf{0}_{[N \times 2]} \\
\mathbf{0}_{[2 M \times 1]} & \boldsymbol{\Phi}_{\boldsymbol{f}[2 M \times 2]}^{t}
\end{array}\right]\left[\begin{array}{c}
T_{f}^{d} \\
\boldsymbol{M}_{\boldsymbol{f}}^{\boldsymbol{d}}
\end{array}\right] \mathrm{d} S .
$$

In Eq.(38), $T_{f}^{d}$ and $\boldsymbol{M}_{\boldsymbol{f}}^{\boldsymbol{d}}$ represent the vertical force and out-of-plane moments prescribed on the boundary $\partial \Omega_{\Sigma}^{e}$. It is worth pointing out that, as far as it concerns the out-of-plane behaviour, the expressions of the stiffness matrix $\boldsymbol{K}_{\boldsymbol{f}}$ and of its sub-matrices obtained for the Cosserat plate element (Eq.(33)) have the same structure and form with those of a thick (Reissner-Mindlin) plate element. Indeed, the present Cosserat FE formulation is more general and encloses the Reissner-Mindlin FE formulation. This latter can be retrieved by a) neglecting the longitudinal shears $\tau_{13}$ and $\tau_{23}$ in the constitutive matrix $\boldsymbol{F}$ (Eq.(20)) and b) by redefining of the operator $\boldsymbol{L}_{\boldsymbol{f} 4}$ in Eq.(18), in order to take into account only the symmetric part of the rotation gradient into the definition of the out-of-plane curvatures $\boldsymbol{\kappa}_{\boldsymbol{f}}$ (see Table 1). In this sense, the FE formulation provided for Reissner-Mindlin plates is a special case of the FE formulation for Cosserat plates presented herein. Due to this similarity, shear locking phenomenon is expected in the limit of very thin geometries in the present Cosserat formulation. This issue will be explored in Section 6.2.

\subsection{Mass matrix}

The mass matrix can be computed by substituting Eq.(22)-(23) into the equation of the kinetic energy $\mathrm{K}^{c}$ (Eq.(11)) calculated for the plate. For the in-plane behaviour this results in the expression:

$$
\mathrm{K}_{m}^{c}=\frac{1}{2}\left[\begin{array}{ll}
\ddot{\boldsymbol{U}}_{\boldsymbol{m}}{ }^{t} & \ddot{\boldsymbol{\Omega}}_{\boldsymbol{m}}{ }^{t}
\end{array}\right]\left[\begin{array}{ll}
\boldsymbol{M}^{\boldsymbol{m}} & \mathbf{0} \\
\mathbf{0} & \Theta^{m}
\end{array}\right]\left[\begin{array}{l}
\ddot{\boldsymbol{U}}_{\boldsymbol{m}} \\
\ddot{\boldsymbol{\Omega}}_{\boldsymbol{m}}
\end{array}\right],
$$

with the mass sub-matrices:

$$
\left[\begin{array}{ll}
\boldsymbol{M}^{\boldsymbol{m}} & \mathbf{0} \\
\mathbf{0} & \boldsymbol{\Theta}^{\boldsymbol{m}}
\end{array}\right]=\int_{\Omega^{e}}\left[\begin{array}{rr}
\boldsymbol{N}_{\boldsymbol{m}[2 N \times 2]}^{t} & \mathbf{0}_{[2 N \times 1]} \\
\mathbf{0}_{[\mathrm{M} \times 2]} & \boldsymbol{\Phi}_{\boldsymbol{m}[M \times 1]}^{t}
\end{array}\right]\left[\begin{array}{rr}
\mathbf{1}_{\boldsymbol{m}[2 \times 2]} & \mathbf{0}_{[2 \times 1]} \\
\mathbf{0}_{[1 \times 2]} & I_{m[1 \times 1]}
\end{array}\right]\left[\begin{array}{rr}
\boldsymbol{N}_{\boldsymbol{m}[2 \times 2 N]} & \mathbf{0}_{[2 \times M]} \\
\mathbf{0}_{[1 \times 2 N]} & \boldsymbol{\Phi}_{\boldsymbol{m}[1 \times M]}
\end{array}\right] \mathrm{d} A .
$$

For the out-of plane behaviour it holds:

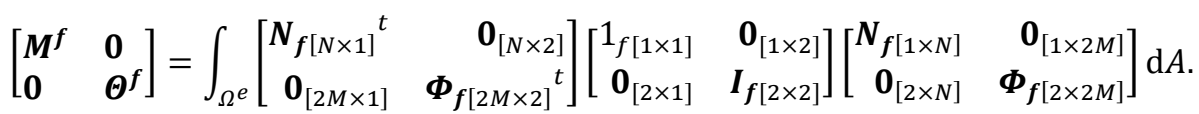

In the present formulation the micro-inertia tensor is considered isotropic. A rigorous way to determine the inertial terms of a Cosserat medium could be that dictated by Germain [47] and Eringen [16] (see also [57]). The mass density $\rho$ may be defined by identification of the rigid microstructure with the material particle of the underlying continuum, as the integral of the mass density distribution $\rho^{\prime}$ over the volume of the particle $V$ :

$$
\rho \mathrm{d} V=\int_{V} \rho^{\prime} \mathrm{d} V^{\prime}
$$

where $[\cdot]^{\prime}$ denotes that the variable is defined at the scale of the particle. Similarly, the micro-inertia tensor can be defined as the second order moment with respect to the position of the centre of the mass of the particle, i.e. [16]:

$$
\rho I_{i j} \mathrm{~d} V=\int_{V} \rho^{\prime} x_{i}^{\prime} x_{j}^{\prime} \mathrm{d} V^{\prime} .
$$


Following the definitions given in this Section, the PDE for a Cosserat plate FE are expressed in matrix form as:

$$
\begin{aligned}
& {\left[\begin{array}{ll}
K_{U U}^{m} & K_{U \Omega}^{m} \\
K_{\Omega U}^{m} & K_{\Omega \Omega}^{m}
\end{array}\right]\left[\begin{array}{l}
U_{m} \\
\Omega_{m}
\end{array}\right]+\left[\begin{array}{ll}
M^{m} & 0 \\
0 & \Theta^{m}
\end{array}\right]\left[\begin{array}{l}
\ddot{U}_{m} \\
\ddot{\Omega}_{m}
\end{array}\right]=\left[\begin{array}{l}
P_{m} \\
Q_{m}
\end{array}\right]} \\
& {\left[\begin{array}{ll}
K_{U U}^{s} & K_{U \Omega}^{s} \\
K_{\Omega U}^{s} & K_{\Omega \Omega}^{s}+K_{\Omega \Omega}^{b}
\end{array}\right]\left[\begin{array}{l}
U_{f} \\
\Omega_{f}
\end{array}\right]+\left[\begin{array}{ll}
M^{f} & 0 \\
0 & \Theta^{f}
\end{array}\right]\left[\begin{array}{l}
\ddot{U}_{f} \\
\ddot{\Omega}_{f}
\end{array}\right]=\left[\begin{array}{l}
P_{f} \\
Q_{f}
\end{array}\right] .}
\end{aligned}
$$

Eq.(44) governs the motion of the element and is to be solved at each step of the analysis. To this purpose, a number of solution methods exist and some of them are exploited in the commercial code Abaqus [58], where the presented formulation has been implemented as a particular User ELement (UEL) and employed within an implicit time-discretization procedure. This makes possible to control the analysis at each step of a prescribed procedure, to compute the stress and deformation state at every Gauss point of the element and to combine the new element solution with that obtained by the pre-existing elements of the code.

\section{The COSS8R element}

The implemented element is an isoparametric quadratic plane rectangle with eight nodes and constant thickness (Fig. 3). Reduced integration is made to avoid shear locking and therefore four Gauss points are used. Each node of the element is equipped with three translational and three rotational DOF. This results in a complete six-degree-offreedom FE formulation, allowing to model shell structures as an assembly of these flat elements [41].

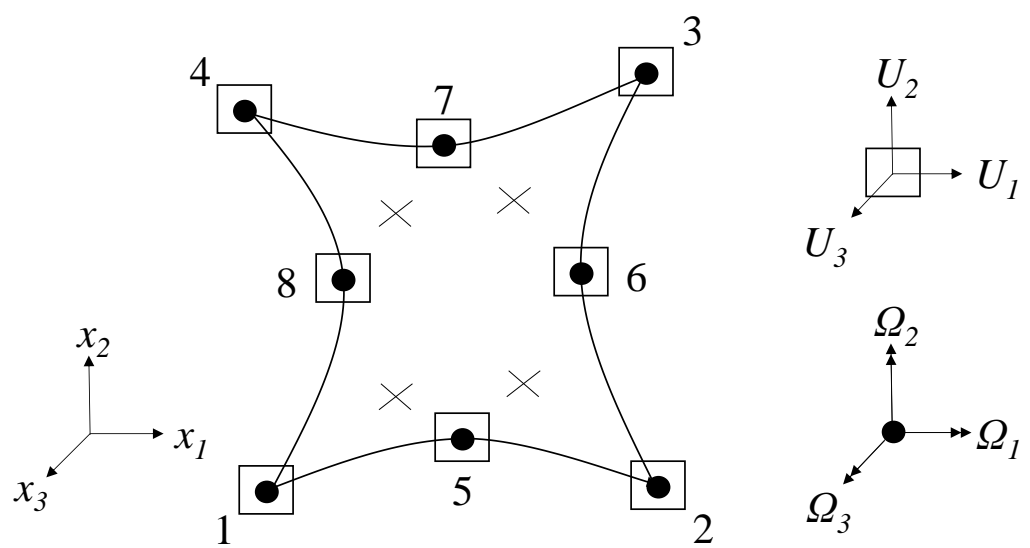

Fig. 3 The COSS8R element, with 4 Gauss points (X), 8 nodes and 6 DOF per node: translations (square) and rotations (circle).

A similar FE formulation has been proposed in literature by [59], but to the author's knowledge its performance has not been yet explored.

\subsection{Choice of the polynomial order of interpolation}

The same quadratic shape functions $\boldsymbol{N}$ and $\boldsymbol{\Phi}$ are used for the polynomial interpolation of the translational and the rotational kinematic fields. This choice is not the only one possible.

An alternative would be to consider Lagrangian elements with linear interpolations for both kinematic fields. However, in this case, Eq.(3)-(4) indicate that some integral terms, as for example the product between the symmetric tensors $\left[\tau_{(i j)} \gamma_{(i j)}\right]$ and the product between the couple stresses and the curvatures $\left[\mu_{i j} \kappa_{i j}\right]$, would be of zero degree (constants). On the other hand, the product between the skew-symmetric tensors $\left[\tau_{[i j]} \gamma_{[i j]}\right]$ would be of second degree. In that case, a selective integration technique should be attempted in order to redress the same level of accuracy in the representation of both deformation measures, since, if only the lower order of interpolation was respected, it would result in a decrease of accuracy for coarse element discretisations.

Another approach could be to choose high precision Hermitian elements, as done by [60]. However, in spite of the higher accuracy reached by this approach, the plate formulation with Hermitian elements would be not trivial and more computationally intensive.

In [56] it is demonstrated that, in the case of an explicitly derived formulation where no numerical integration is involved, a triangular Cosserat element for plane elasticity that uses quadratic shape functions for both displacements 
and rotations produces excellent results if compared to linear interpolations. Only marginally better would be the behavior of elements with quadratic (for translations) and linear (for rotations) approximations [56].

Indeed, the adoption of quadratic shape functions for the displacement field and of linear shape functions for the rotational field would assure the same order of interpolation, as far as it concerns the in-plane strain vector $\boldsymbol{\gamma}_{\boldsymbol{m}}$. This as consequence of its definition (Eq.(1)). Nevertheless, the adoption of linear functions for the rotations would lead, after Eq.(2), to constant in-plane $\boldsymbol{\kappa}_{\boldsymbol{m}}$ and out-of-plane $\boldsymbol{\kappa}_{\boldsymbol{f}}$ curvatures within the element. In view of the representation of flexural problems, this is considered as a limitation.

In order to overcome this problem, we make use of quadratic shape functions for both the translational and the rotational fields over the FE. Such a formulation allows to cover the interpolation order given by a linear/quadratic formulation and to increase the precision with respect to the curvatures. Aiming at modelling both the in-plane and the out-of-plane behaviour, this choice is expected to provide a good level of accuracy in case of coarse meshes and be still advantageous in view of the dynamic analyses, where the use of too refined formulations would increase excessively the computational cost.

\subsection{Zero-energy modes investigation}

Reduced integration comes with the price of introducing modes of deformation with zero energy, i.e. modes for which the element does not exhibit any stiffness [61]. In the case of classical quadrilateral membrane elements such modes take the shape of an hourglass and they have to be controlled in order to assure accurate results. One way consists, for instance, in introducing an additional drilling rigidity into the element stiffness matrix [41]-[43]. This artificial stiffness does not have a particular physical meaning except in the framework of a Cosserat continuum, where it is provided by the in-plane particle rotations.

\section{HOURGLASS MODES}

In a general manner we consider the following standard eigenproblem:

$$
[\boldsymbol{K}-\lambda \mathbf{1}] \overline{\boldsymbol{U}}=0 .
$$

The solution of Eq.(45) gives L eigenvalues $\lambda_{l}$ and L nodal eigenvectors $\overline{\boldsymbol{U}}^{l}$, with $l=1, \ldots, \mathrm{L}$ and $\mathrm{L}$ being the dimension of the considered local stiffness matrix $\boldsymbol{K}$. It is known that every eigenvalue is equal to twice the strain energy due to the displacement field provided by the associated eigenvector [62]. As a consequence, one may expect from the solution of the eigenproblem as many null eigenvalues as the number of rigid motions and, for each additional null eigenvalue, an associated non-rigid motion with zero deformation energy can be found. In computational terms, this indicates a matrix rank deficiency. In mechanical terms, this shows that the formulation is affected by the presence of zero-energy modes.
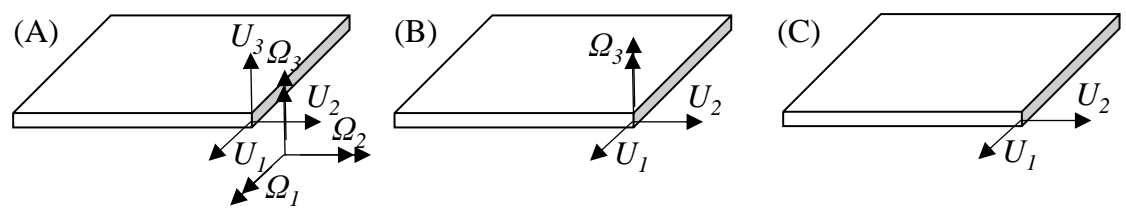

Fig. 4 Elements tested for zero-energy modes investigation: (A) Cosserat element, plate configuration; (B) Cosserat element, membrane configuration; (C) classical Cauchy element, membrane part.

Starting from the present Cosserat formulation, we calculate the eigenvalues of the stiffness matrix $\boldsymbol{K}$ for three different cases (Fig. 4). First, the eigenproblem is solved for a complete six-degree-of-freedom Cosserat assembly (Fig. 4-(A)). The same investigation is made by considering only the membrane behaviour of the Cosserat element (Fig. 4-(B)). Then, only the in-plane translations are kept, reducing the present formulation to a classical Cauchy membrane element (Fig. 4-(C)). The stiffness matrix considered in each case is one (or an assembly) of the submatrices defined in Section 4.1. For the case (C) we also impose symmetry into the in-plane shear stress in order to retrieve Cauchy continuum. As shown in Appendix 2, this can be accounted for in the construction of the material matrix $\boldsymbol{A}$, by respecting the following identities:

$$
A_{1212}=A_{2121}=A_{1221}=A_{2112} \text {. }
$$

In Table 2, we give the comparison between the three aforementioned configurations, in terms of the number of computed zero-energy modes. Despite the reduced integration, the COSS8R element does not show any rank deficiency. 
The origin of the zero-energy modes can be found in the difference between the number of local deformation variables of the continuum model that we intend to discretise and the number of deformation modes of the actual numerical model. Hence, given the continuum, it is sufficient to increase the number of degrees of freedom, for example through mesh refinement or by increasing the interpolation order, in order to alleviate the matrix deficiency and make the zero-energy mode non-communicable [61]. There exist, however, certain situations in which the problem still occurs.
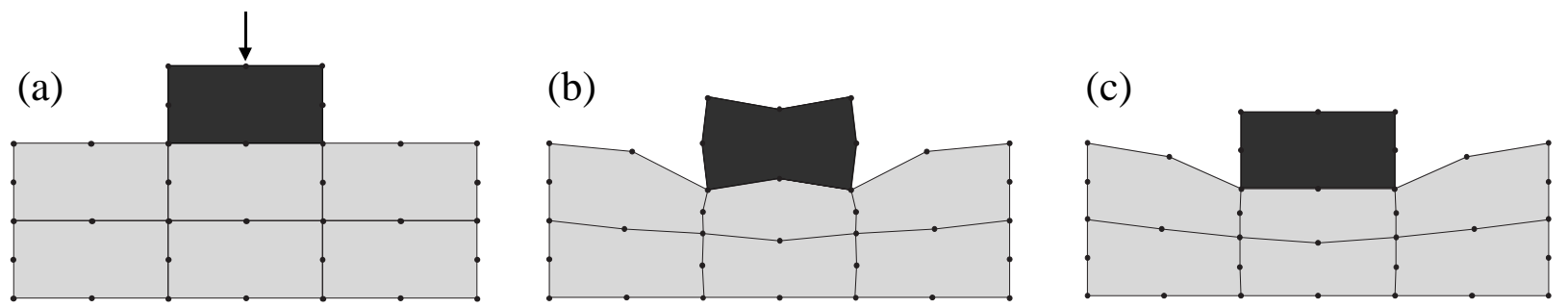

Fig. 5 (a) a highly rigid element resting upon a layer of flexible elements with reduced integration; (b) propagating spurious mode in an assembly of Cauchy (S8R) elements; (c) accurate results from a COSS8R subdivision.

Herein we consider the well-discussed plane problem of Fig. 5 (see [41]). Because of the reduced integration, an hourglass mode is activated in the rigid element as a result of the applied force, and it gives rise to a spurious (or communicable) mode which is able to propagate within a certain area in the elements below. In the case of the classical S8R element (Fig. 5-(b)), only the use of a complete integration would allow to overcome such problem and give accurate solutions, with the price of shear locking behaviour. On the contrary, the COSS8R element does not have this problem, as the rotational DOF perform an intrinsic action of hourglass control (Fig. 5-(c)).

It is interesting to investigate how these spurious modes become manifest. To this end, we consider a homogeneous isotropic Cosserat centrosymmetric material. Matrices $\boldsymbol{A}$ and $\boldsymbol{D}_{\boldsymbol{m}}$ in Eq.(19) are expressed as:

$$
\boldsymbol{A}=\left[\begin{array}{cccc}
K+G & K-G & 0 & 0 \\
K-G & K+G & 0 & 0 \\
0 & 0 & G+G_{c} & G-G_{c} \\
0 & 0 & G-G_{c} & G+G_{c}
\end{array}\right] \quad \boldsymbol{D}_{\boldsymbol{m}}=\left[\begin{array}{cc}
2 G l_{c} & 0 \\
0 & 2 G l_{c}
\end{array}\right]
$$

where $K$ is the compression modulus, $G$ is the shear modulus, $G_{c}$ is the Cosserat shear modulus and $l_{c}$ denotes the internal length under shear. It is useful to define $\alpha=G_{c} / G$ as the coupling factor. As we show in Appendix 2, this parameter allows to control the magnitude of the skew-symmetric term of the membrane strain vector, i.e. $\gamma_{[12]}$. For $\alpha=0$, in-plane rotations are annihilated and the classical Cauchy element is retrieved through Eq.(46) (Fig. 5(b)). For $\alpha=1$, the COSS8R element is recalled (Fig. 5-(c)). Intermediate values of $\alpha$ allows to control the magnitude of, say, the Cosserat's term $\gamma_{[12]}$.

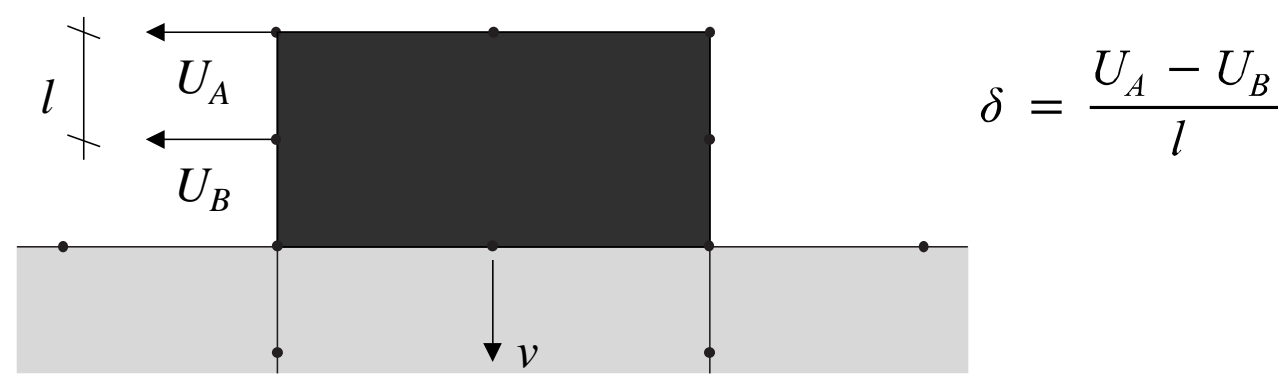

Fig. 6 Definition of the distorsion $\delta$ of the rigid element.

Always with reference to the above example (Fig. 5), a parametric analysis is carried out on $\alpha$. The results are presented in Fig. 7, in terms of the distortion $\delta$ and the displacement $v$, which are defined in Fig. 6 and normalized with the values calculated for $\alpha=0$. Fig. 7 shows an immediate decrease of distortion due to the introduction of the Cosserat's term (Fig. 7-left). Moreover, the exact solution is reached rapidly by varying the coupling factor $\alpha$ from 0 to 1 (Fig. 7-right). Therefore we can defend the idea that the non-symmetry of the stress tensor permits to avoid the rank deficiency into the element membrane stiffness in case of reduced integration. The non-symmetry of the stress tensor is a key feature of Cosserat continuum. 

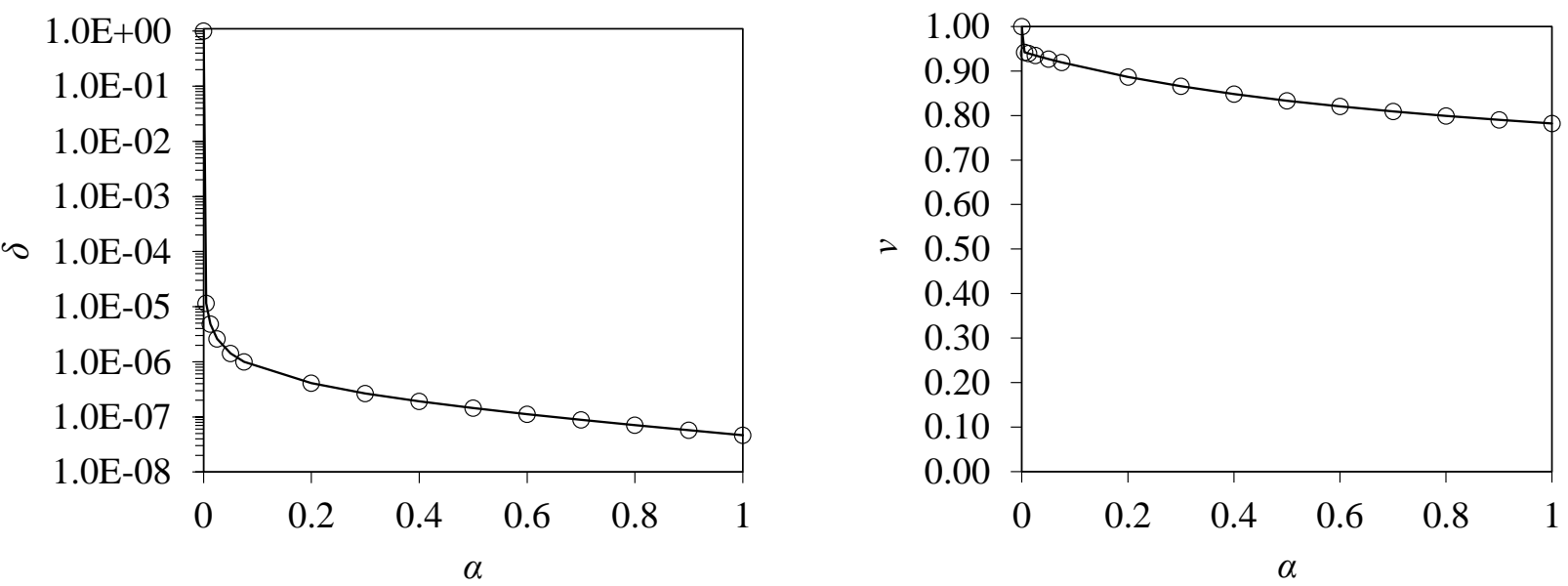

Fig. 7 Normalised distortion factor (left) and normalised vertical displacement (right) versus the coupling factor $\alpha$. The classical Cauchy (S8R) element is retrieved for $\alpha=0$. The COSS8R element is for $\alpha=1$.

\section{STIFFNESS MATRIX DEFICIENCIES IN MODELLING SHELL STRUCTURES}

Regarding the complete plate formulation and the possibility to model shell spatial structures, we demonstrate in Appendix 3 that the coupling factor $\alpha$ enters the definition of the first term of the stiffness sub-matrix (31). Such matrix, as it is indicated by [44], has also a stabilization effect in the modelling of shell structures by assembling flat plate elements. We can thus conclude on the good computational performance of the present Cosserat plate element. The non-symmetry of the stress tensor has in fact a general rank stabilization effect versus the occurrence of hourglass modes in membrane behaviour, and matrix deficiencies in shell structures assemblies. This is a clear advantage as compared to classical-continuum-based FE formulations, where the use of artificial hourglass control techniques is inevitable.

\subsection{Consistent mass matrix}

The dynamic counterpart of Eq.(45) is the generalized eigenproblem:

$$
[\boldsymbol{K}-\lambda \boldsymbol{M}] \overline{\boldsymbol{U}}=0 .
$$

Always employing a reduced integration for the computation of the stiffness matrix $\boldsymbol{K}$ (the case (A) of Fig. 4 is herein referred to), reduced and complete integration are performed for the computation of the local mass matrix $\boldsymbol{M}$. Generalized eigenvalue analyses are carried on the aforementioned matrices and reveal the occurrence of infinite eigenvalues, in the case of reduced integration of the mass matrix. From a mechanical point of view, this corresponds to the presence of massless degrees of freedom [63], for which the system exhibits infinite stiffness. A static condensation would then be necessary on such variables in order to give accurate results, and the element would result in lumped mass components. Therefore complete integration is used over the mass matrix, so as to keep an effectively consistent formulation.

Through this method we also confirm the absence of zero-energy modes in the dynamic regime.

\section{$6 \quad$ Element validation}

A number of numerical tests and examples are presented in this Section with the goal of assessing the performance of the present COSS8R element both in static and dynamic regime. In particular, a patch test specifically designed for Cosserat continua is made to prove the efficiency of the element in representing homogeneous in-plane relative strain and curvature states. Shear locking investigation is then carried out to attest the out-of-plane response of the element in the limit of thin geometries. The wave dispersion functions are finally calculated with reference to a onedimensional problem and compared to the analytical solution.

\subsection{Patch test}

We consider the rectangular region of Fig. 8, where an internal element is introduced within the patch in order to induce a geometric distortion to the whole discretization. 

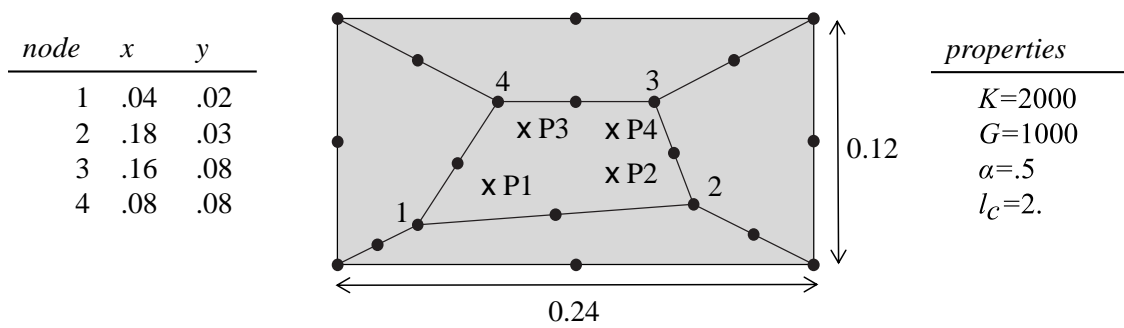

Fig. 8 Element discretization employed for the patch test.

The patch test used here is inspired by [56], who used it for triangular elements, and it is adequately modified for rectangular FE. A homogeneous isotropic linear elastic Cosserat material is taken into account. The material matrix given by Eq.(47). Three tests are performed dealing with the membrane (or in-plane) behaviour [56], see Table 3. Test 1 verifies that the element is capable of reproducing constant symmetric shear components, as in a classical formulation. In Test 2, a non-symmetric constant shear is imposed through the application of external couples $m_{3}$. For Test 3 , the solution is such that the in-plane couple stresses are identical and constant.

The boundary conditions which allow the situations above to be achieved are applied to the external nodes of the patch and the solution is checked at Gauss point $P 1$ of the internal element (Fig. 8). As shown in Table 4, good agreement with the theoretical solution is achieved in all tests. In Test 3, the level of accuracy reached by the formulation is comparable with that found by [56] for their quadratic triangular element MQUAT.

\subsection{Shear locking investigation}

We consider the example of simply-supported and clamped-edge $L \times L$ square plates of constant thickness $h$, subjected to uniform distributed load $p$ (Fig. 9). In order to investigate the shear locking phenomenon, several analyses are carried out on these configurations by considering various FE subdivisions of increasing $L / h$ ratios.
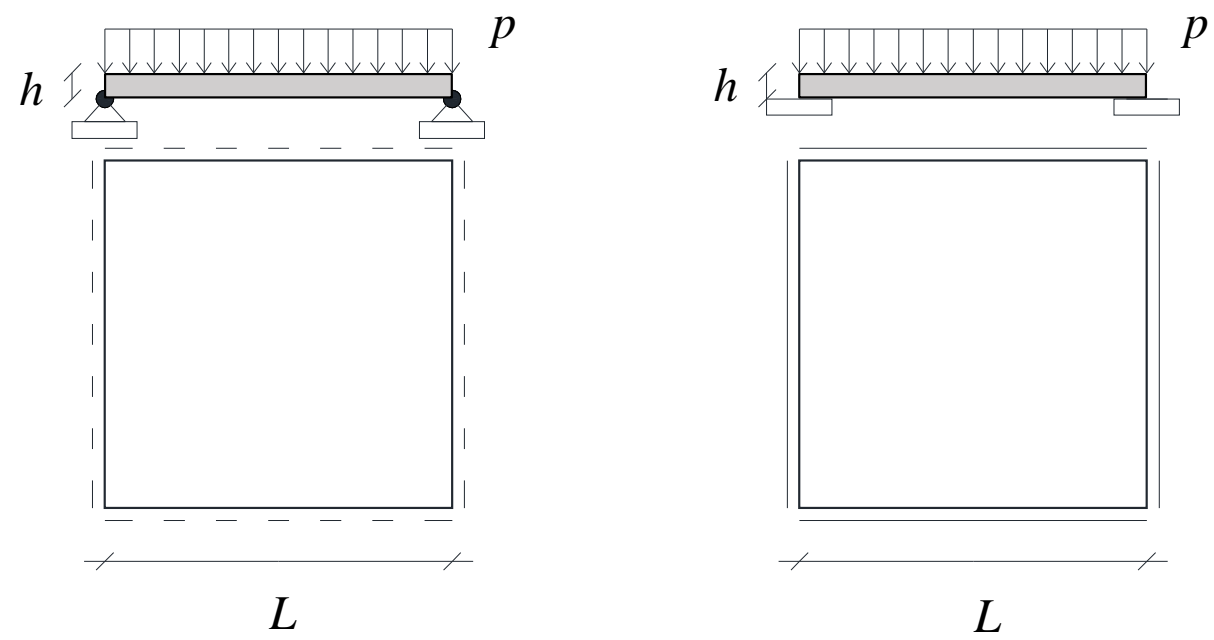

Fig. 9 Bending-plate in two configurations: simply supported (left) and clamped edges (right).

To this purpose, we take into account the constitutive law proposed by Altenbach \& Eremeyev [53], who, starting from a Cosserat 3D flat configuration, deduce an isotropic relation for micropolar plates by integration in the thickness direction. Matrices $\boldsymbol{F}$ and $\boldsymbol{D}_{\boldsymbol{f}}$ in Eq.(20) yield:

$$
\boldsymbol{F}=\left[\begin{array}{cccc}
0 & 0 & 0 & 0 \\
0 & \alpha_{4} & 0 & 0 \\
0 & 0 & 0 & 0 \\
0 & 0 & 0 & \alpha_{4}
\end{array}\right] \quad \boldsymbol{D}_{\boldsymbol{f}}=\left[\begin{array}{cccc}
\beta_{1}+\beta_{2}+\beta_{3} & \beta_{1} & 0 & 0 \\
\beta_{1} & \beta_{1}+\beta_{2}+\beta_{3} & 0 & 0 \\
0 & 0 & \beta_{3} & \beta_{2} \\
0 & 0 & \beta_{2} & \beta_{3}
\end{array}\right],
$$

where the parameters $\alpha_{4}, \beta_{1}, \beta_{2}, \beta_{3}$ are here retrieved from the works of Lakes made on high-density rigid polyurethane closed-cell foams (see Table 2-PU in [53]). 

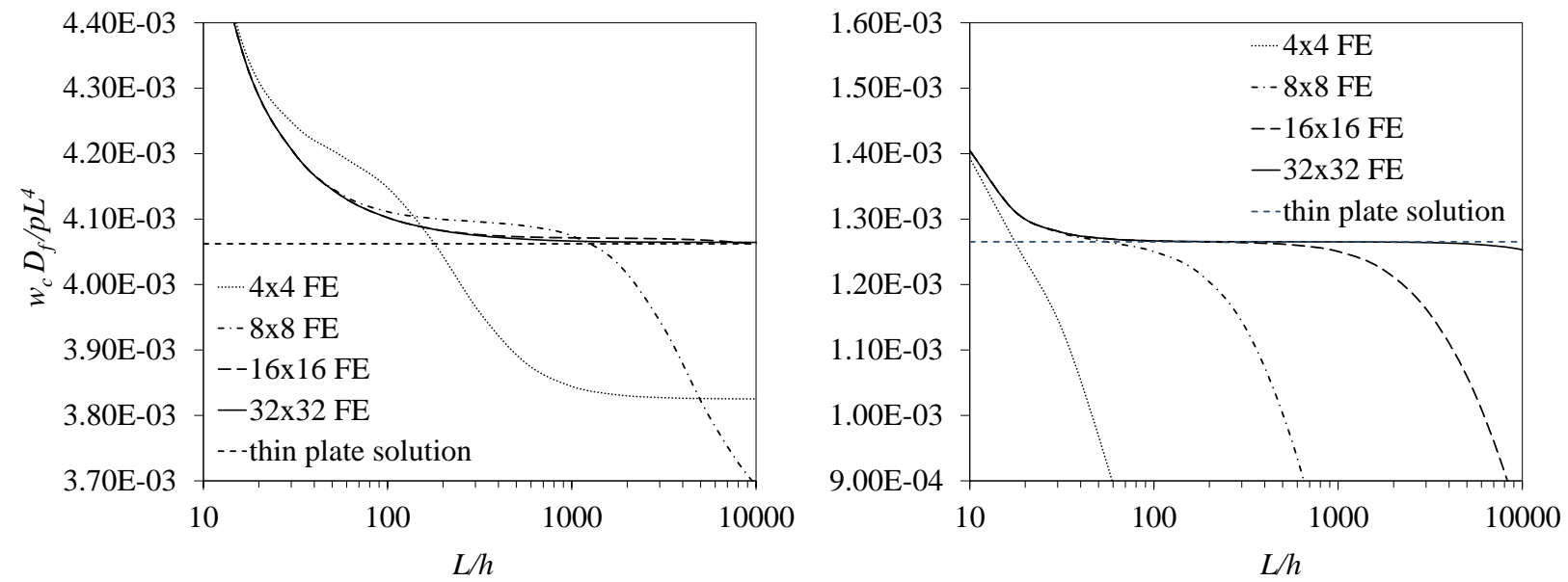

Fig. 10 Normalised central deflection versus length-to-thickness ratio for given meshes. Left: simply supported plate. Right: clamped-edge plate.

In Fig. 10 we show the resultant deflection $w_{c}$, measured at the centre of the plate. The out-of-plane bending rigidity used for normalization is $D_{f} \equiv \beta_{3}$. The results are qualitatively similar to those found in literature for the thick (Reissner-Mindlin) plate elements (see [41]). In particular, the performance of the element reduces as the geometry of the plate becomes thin, and this phenomenon is more apparent for the plate with clamped edges than for the simply supported one. Nevertheless, in both cases convergence is guaranteed upon mesh refinement. It is to note that, for high $L / h$ ratios, the structural response reaches the solution predicted by the thin (Love-Kirchhoff) plate theory (see [64]). This can be explained by the fact that, as for the Reissner-Mindlin formulation, the limit of thin geometries plays the role of an internal kinematical constraint, for which the transverse shear deformations tend to vanish. Moreover, due to the symmetry of the problem and the constitutive law considered, the torsional response is symmetric in the plate directions. These two conditions allow to retrieve the kinematics of a thin plate, in the limit of $L / h \rightarrow \infty$.

\subsection{Dispersion functions}

We consider a narrow infinite strip of Cosserat material in the $x_{2}$-direction, invariant in the $x_{1}$ and $x_{3}$ directions. The flexural problem of this one-dimensional configuration is governed by the following PDE (Eq.(12)):

$$
\begin{aligned}
& \tau_{32,2}-\rho \ddot{u}_{3}=0 \\
& \mu_{12,2}+\tau_{32}-\rho I_{1} \ddot{\omega}_{1}=0 .
\end{aligned}
$$

By using the definition of the deformation measures (Eq.(1)-(2)) and the constitutive relations (Eq.(20)) one obtains:

$$
\begin{aligned}
& F^{3232}\left[u_{3,2}-\omega_{1}\right]_{, 2}-\rho \ddot{u}_{3}=0 \\
& D_{f}^{1212} \omega_{1,22}+F^{3232}\left[u_{3,2}-\omega_{1}\right]-\rho I_{11} \ddot{\omega}_{1}=0 .
\end{aligned}
$$

which has indeed the same structure of that governing the free-vibrations of a Timoshenko beam. Setting:

$$
\begin{gathered}
u_{3}=A \mathrm{e}^{\mathrm{i}\left(\kappa x_{2}+v t\right)} \\
\omega_{1}=B \mathrm{e}^{\mathrm{i}\left(\kappa x_{2}+v t\right)},
\end{gathered}
$$

in which $\kappa$ is the wave number, $v$ denotes the angular frequency, and $A, B$ are imaginary constants and by substituting Eq.(52) into Eq.(51), we obtain, in matrix notation:

$$
\left[\begin{array}{ll}
-F^{3232} \kappa^{2}+\rho v^{2} & -\mathrm{i} F^{3232} \kappa \\
\mathrm{i} F^{3232} \kappa & -F^{3232}-D_{f}^{1212} \kappa^{2}+I_{1} \rho v^{2}
\end{array}\right]\left[\begin{array}{l}
A \\
B
\end{array}\right]=\left[\begin{array}{l}
0 \\
0
\end{array}\right] .
$$

The above system admits non-trivial solutions only when:

$$
\kappa^{4}-\left(\frac{\rho I_{11}}{D_{f}^{1212}}+\frac{\rho D_{f}^{1212}}{F^{3232}}\right) \kappa^{2} v^{2}-\frac{\rho}{D_{f}^{1212}} v^{2}+\frac{\rho^{2} I_{11}}{F^{3232} D_{f}^{1212}} v^{4}=0 .
$$

The roots of Eq.(54) are called dispersion relations and they can be expressed in a form similar to [24] as follows: 


$$
v_{1,2}(\kappa)=\sqrt{-\frac{\beta(k)}{2 \alpha(k)} \pm \sqrt{\left[\frac{\beta(k)}{2 \alpha(k)}\right]^{2}-\frac{\gamma(k)}{\alpha(k)}}}
$$

where

$$
\begin{aligned}
& \alpha(\kappa)=\rho^{2} I_{1} \\
& \beta(\kappa)=-\left(\rho \kappa^{2} I_{1} F^{3232}+D_{f}^{1212} \rho \kappa^{2}+F^{3232} \rho\right) \\
& \gamma(\kappa)=D_{f}^{1212} F^{3232} \kappa^{4} .
\end{aligned}
$$

Similar to the Timoshenko beam theory, the dispersion relations reveal the two basic oscillation modes of the system. With reference to Fig. 11 we have: mode 1, the micro-rotation mode, describing the pure bending shape, and mode 2 , the shear deformation mode, representing a shear strain state. The superposition of such modes gives the flexural response of the beam.

The membrane problem of the aforementioned one-dimensional Cosserat configuration is governed by the following PDE:

$$
\tau_{22,2}-\rho \ddot{u}_{2}=0 .
$$

By using again Eq.(1)-(2) and (19), one can rewrite the above equation as:

$$
A^{2222} u_{2,22}-\rho \ddot{u}_{2}=0 .
$$

Looking for a solution of the form similar to Eq.(52), we obtain the following linear relation:

$$
v_{3}(\kappa)=\sqrt{\frac{A^{2222}}{\rho}} \kappa
$$

which is associated to a mode 3 of longitudinal waves.

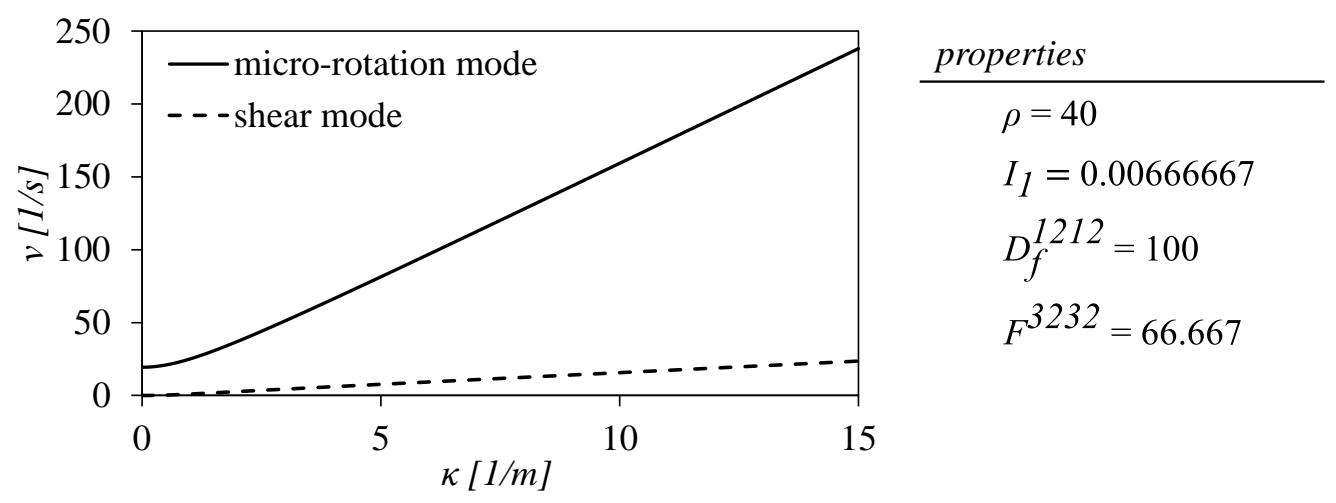

Fig. 11 Dispersion relations for a one-dimensional Cosserat flexural problem: micro-rotation mode $v_{1}$ and shear strain mode $v_{2}$.

For validation purposes, we consider as illustrative example a simply supported beam of length $L$. In such case, the resulting wave numbers are:

$$
\kappa_{n}=\frac{n \pi}{L}, \text { with } n=1,2,3, \ldots
$$

corresponding to wave lengths:

$$
\lambda_{n}=\frac{2 \pi}{\kappa_{n}}=\frac{2 L}{n}, \text { with } n=1,2,3, \ldots .
$$

Once the modal wave numbers are calculated, it is necessary to pass by the dispersion relations (Fig. 11) to obtain the corresponding natural frequencies.

The beam is discretized by a series of 20, 40, 80 and 160 FE over its length. Natural frequencies are computed by a modal analysis based on Lanczos method and compared with the analytical values indicated above. The relative error committed by the FE in representing the longitudinal vibrations is zero even with the coarsest discretization. The relative error committed in the evaluation of the first eleven flexural oscillation modes is plotted in Fig. 12, versus the normalised wave length $\lambda_{n} / L$. A 9-digit precision is used for the wave lengths. The relative error is defined as: 


$$
\epsilon_{n}^{\lambda}=100 \times \frac{\lambda_{n}-\lambda_{n}^{F E}}{\lambda_{n}} .
$$

In general, we may note how the convergence to the analytical values is achieved by the COSS8R element upon mesh refinement. As expected, we observe that, for a given discretization, the relative error decreases for increasing wave lengths. Therefore, a quite fine mesh is needed to extract the highest frequencies from the FE model: a 40-element subdivision is considered acceptable.

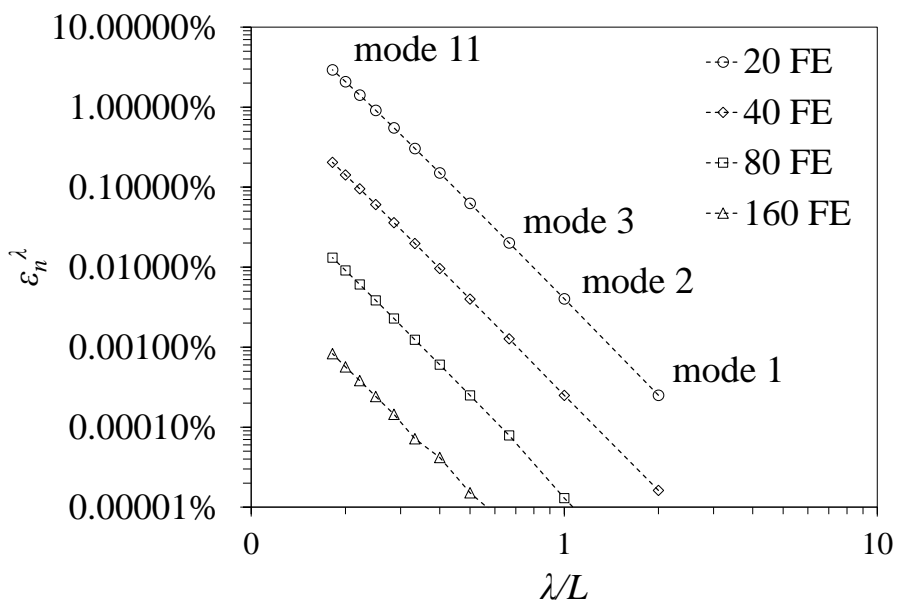

Fig. 12 Relative error committed in the evaluation of the first eleven flexural wave lengths.

\section{$7 \quad$ Application to masonry structures}

The purpose of this Section is to highlight the practical interest of the above Cosserat FE formulation in view of engineering applications. The case of masonry panels is selected, due to the apparent microstructure of such material. Through illustrative examples, we investigate the performance of the COSS8R element for the prediction of the modal response of masonry panels, considered as equivalent Cosserat plates. In particular, we focus on the specific role played by the drilling rotations.

For the analyses, we adopt the homogenized constitutive law for Cosserat plates proposed by Stefanou et al. [8]. This model is derived by identification of a periodic lattice made of regular rigid building blocks with an equivalent 3D Cosserat continuum. The resulting model is an equivalent micropolar plate with orthotropic properties, which accounts for both the in- and the out-of-plane deformation of masonry in the dynamic regime. For the expression of the matrices for the constitutive laws and the inertial terms, the reader is referred to the original work [8].
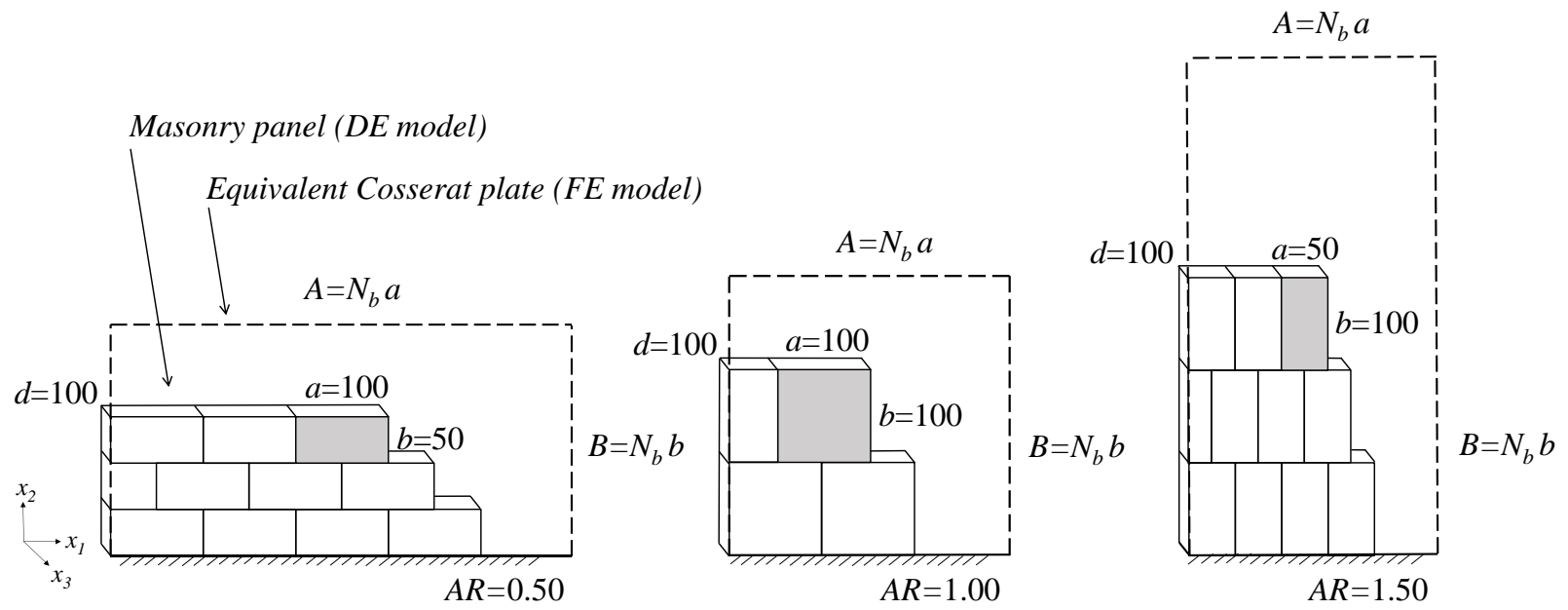

Fig. 13 The three simulated masonry panels modelled with DE and with equivalent Cosserat plates by the COSS8R element: notation, boundary conditions and aspect ratios $A R=B / A$. 
Modal analyses are carried out on plates having 3 different aspect ratios $A R=B / A$ (see Fig. 13). For each of these three configurations, several simulations are launched by increasing the number of blocks $N_{b}$ that compose the panels in both the horizontal and the vertical direction. This operation is done simultaneously, so that, for every given simulation, the panel's aspect ratio is kept constant and equal to the blocks' aspect ratio: $B / A=b / a$. The scale ratio, that is defined herein as the ratio between the size of the blocks and the size of the panel, decreases with the inverse of the number of blocks disposed in each direction, i.e. $a / A=b / B=1 / N_{b}$. On the contrary, the length-to-thickness ratio (representing the slenderness of the panel) increases as $B / d=N_{b} b / d$ (the thickness of the panel is kept constant). This is exactly equivalent with keeping fixed the overall size of the panel and reducing the size of the blocks, without varying their shape.

\subsection{Comparison with Discrete Elements solution}

The Cosserat homogenized constitutive law for masonry [8] is implemented in the COSS8R element and incorporated in Abaqus through the subroutine UEL. The results produced by FE are compared with those obtained from the use a Discrete Elements (DE) analogue model. This latter is created by means of the numerical package 3DEC ([65], [66]). The analogue DE model consists in an assemblage of rigid blocks, which are disposed as presented in Fig. 13. The blocks interact with the adjacent blocks through linear elastic interfaces, that are governed by the same coefficients used in the homogenization model considered in [8]. In this way, the same assumptions are made as far as it concerns the lattice studied by [8], and, consequently, no calibration is needed between the DE and Cosserat FE model.

Modal analyses are carried out on the analogue DE model using the same strategy as described above for the FE model: the number of blocks is progressively increased, while the thickness of the assembled masonry panel is kept constant. It is worth mentioning that, according to [67], a large number of contact-points are required across the thickness of the panel in order to obtain an accurate representation of the out-of-plane behaviour with the DE model. This remarkably increases the calculation cost of the DE model, to FE's advantage, for which a 8x8-FE discretization is considered sufficient after having performed a mesh convergence analysis (see Section 6.3).

The first in-plane and the first two out-of-plane modes, corresponding respectively to shear, bending and torsion oscillation modes are extracted from the COSS8R (Abaqus) and the DE analogue model (3DEC). The natural frequencies for each mode are compared in Fig. 14-Fig. 16 in function of the number of bundling blocks $N_{b}$ composing the panel in both directions (Fig. 13). We observe that the frequencies provided by FE are slightly lower than those produced by DE, and that, for increasing number of blocks, DE and FE converge.
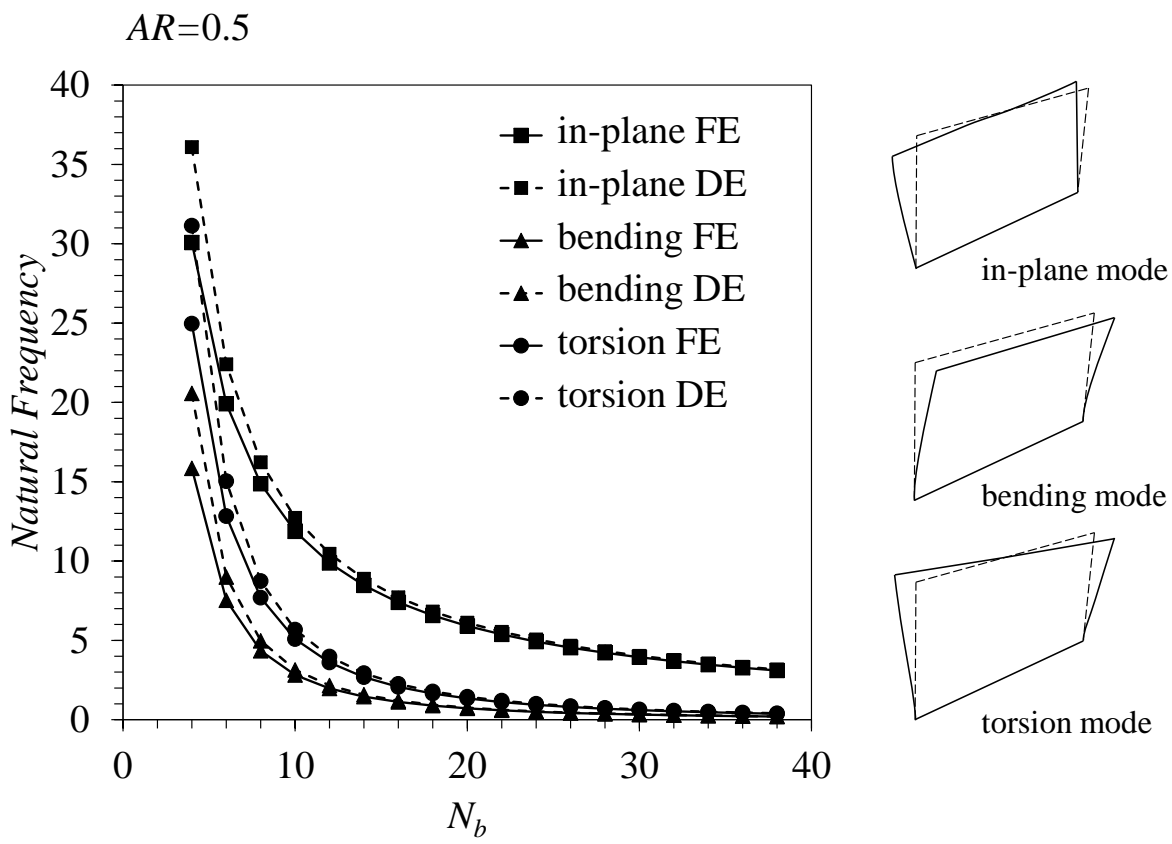

Fig. 14 Modal frequencies of the masonry panel versus the number of building blocks composing the panel in both directions. Comparison between the results extracted by DE and by the use of the equivalent Cosserat plate model for masonry implemented in a COSS8R FE discretisation. Results for aspect ratio $A R=0.50$. 

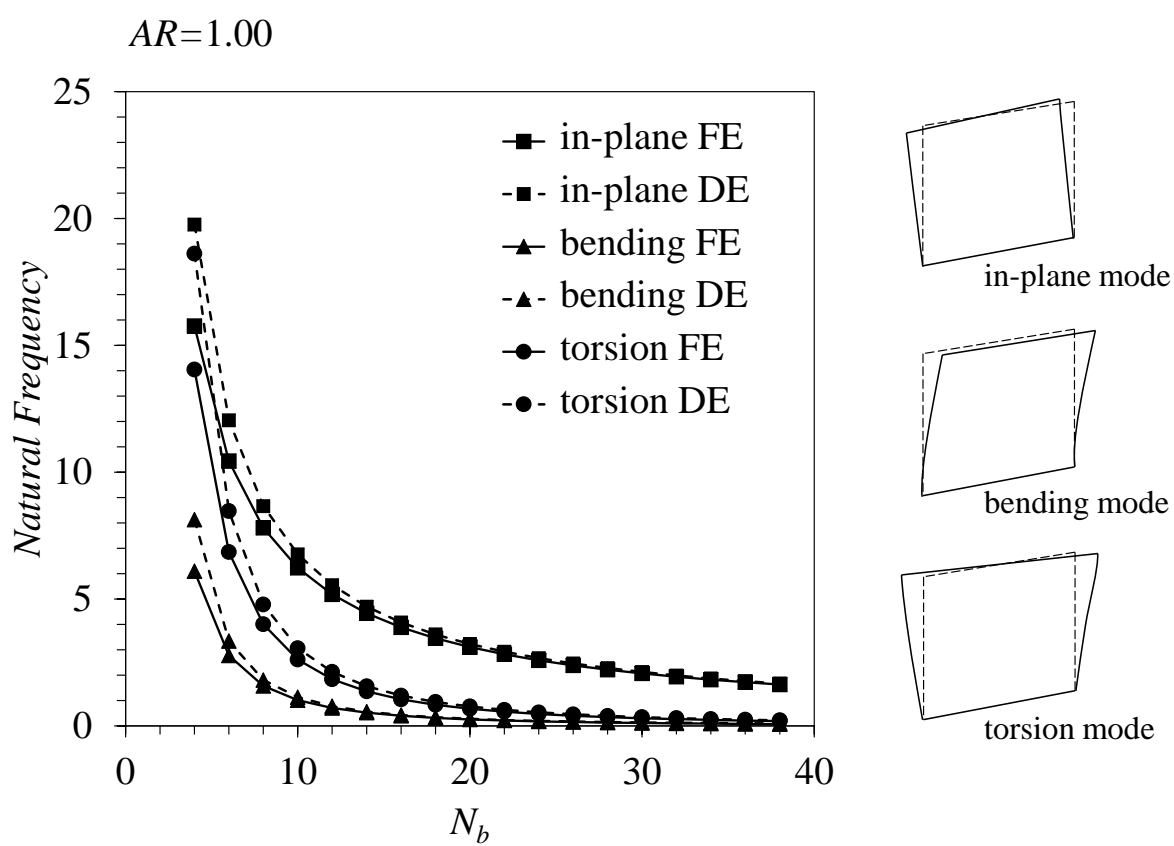

Fig. 15 Modal frequencies of the masonry panel versus the number of building blocks composing the panel in both directions. Comparison between the results extracted by DE and by the use of the equivalent Cosserat plate model for masonry implemented in a COSS8R FE discretisation. Results for aspect ratio $A R=1.00$.
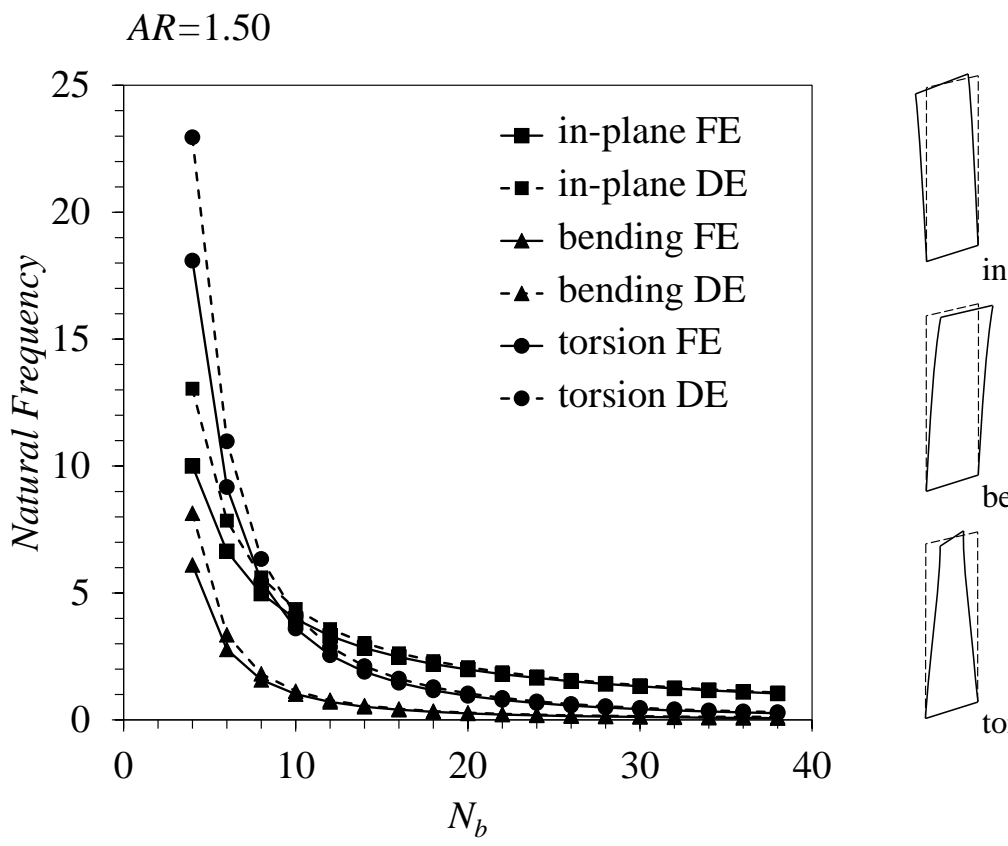

in-plane mode
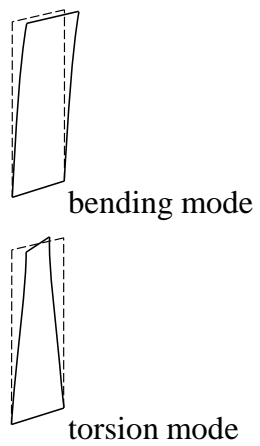

Fig. 16 Modal frequencies of the masonry panel versus the number of building blocks composing the panel in both directions. Comparison between the results extracted by DE and by the use of the equivalent Cosserat plate model for masonry implemented in a COSS8R FE discretisation. Results for aspect ratio $A R=1.50$.

\subsection{The role of drilling rotations}

In Fig. 17 we present the percentage of the modal effective mass related to the first in-plane deformation mode of the masonry panels. The modal effective mass associated to the horizontal and the vertical translations $u_{1}, u_{2}$ is computed over the element in the following manner [58]:

$$
\overline{\mathcal{M}}_{1}=\frac{\left(\overline{\boldsymbol{U}}_{\boldsymbol{m}}{ }^{t} \boldsymbol{M}^{m} \boldsymbol{\Delta}_{\mathbf{1}}\right)^{2}}{\overline{\boldsymbol{U}}_{\boldsymbol{m}}{ }^{t} \boldsymbol{M}^{m} \overline{\boldsymbol{U}}_{\boldsymbol{m}}} \quad \overline{\mathcal{M}}_{2}=\frac{\left(\overline{\boldsymbol{U}}_{\boldsymbol{m}}{ }^{t} \boldsymbol{M}^{m} \boldsymbol{\Delta}_{2}\right)^{2}}{\overline{\boldsymbol{U}}_{\boldsymbol{m}}{ }^{t} \boldsymbol{M}^{m} \overline{\boldsymbol{U}}_{\boldsymbol{m}}} .
$$


The present Cosserat formulation also allows the calculation of the modal effective moment of inertia over each finite element. This effective inertia is associated to the drilling rotations of the plate $\omega_{3}$, through the expression:

$$
\overline{\mathfrak{I}}_{3}=\frac{\left(\overline{\boldsymbol{\Omega}}_{\boldsymbol{m}}{ }^{t} \boldsymbol{\Theta}^{m} \boldsymbol{\Delta}_{3}\right)^{2}}{\overline{\boldsymbol{\Omega}}_{\boldsymbol{m}}{ }^{t} \boldsymbol{\Theta}^{m} \overline{\boldsymbol{\Omega}}_{\boldsymbol{m}}} .
$$

In the above, $\overline{\boldsymbol{U}}_{\boldsymbol{m}}$ and $\overline{\boldsymbol{\Omega}}_{\boldsymbol{m}}$ denote the in-plane nodal translations and rotations composing the eigenvector associated to the oscillation mode considered. The element mass sub-matrices are calculated according to Eq.(40). The vectors $\Delta_{i}$ give the response of every DOF of the element to a unitary motion applied in the $i$-th direction, which consists in a displacement (for $i=1,2$,) and in an axis rotation (for $i=3$ ). Note that the modal effective masses $\overline{\mathcal{M}}_{1}, \overline{\mathcal{M}}_{2}$ and the moment of inertia $\overline{\mathfrak{I}}_{3}$ are computed by Abaqus for the whole element discretisation. In Fig. 17 we present the effective mass and inertia moment normalized, respectively, by the total mass $\mathcal{M}_{\mathrm{TOT}}$ and the total in-plane moment of inertia $\mathfrak{I}_{\text {TOT }}$ of the plate:

$$
\begin{aligned}
& \mathcal{M}_{\mathrm{TOT}}=\rho A B d=\rho N_{b}^{2} a b d \\
& \widetilde{\Im}_{\mathrm{TOT}}=\frac{\mathcal{M}_{\mathrm{TOT}}}{12} \rho\left(A^{2}+B^{2}\right) d=\frac{\mathcal{M}_{\mathrm{TOT}}}{12} \rho N_{b}^{2}\left(a^{2}+b^{2}\right) d .
\end{aligned}
$$

We observe that the amount of the modal inertia that is taken by the drilling rotations for the first in-plane mode increases remarkably with the aspect ratio of the panel and with elongated blocks. In particular, for aspect ratios higher than the unit, this quantity amounts to approximately $50 \%$ of the total rotary inertia (see Fig. $17, A R=1.50$ ). The rest of the effective modal mass (until 100\%) is covered by other higher frequency membrane modes that are not examined herein. This suggests that the drilling rotations can have a significant participation in the inertial response of masonry panels.

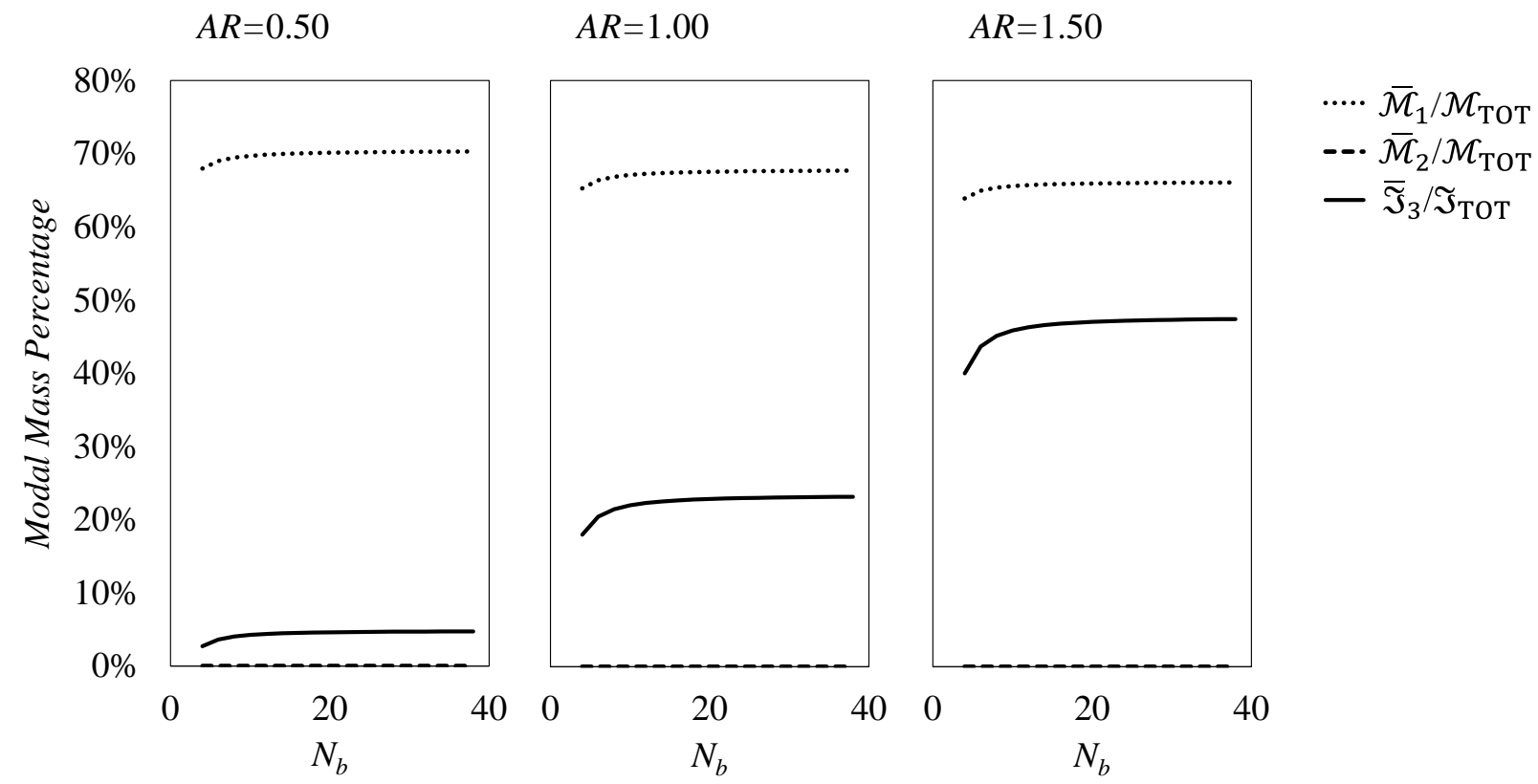

Fig. 17 Percentage of modal effective masses and moment of inertia for the first in-plane mode of the masonry panel, versus the number of building blocks composing the panel in both directions. The modal masses $\overline{\mathcal{M}}_{1}$ and $\overline{\mathcal{M}}_{2}$ are associated to the horizontal and the vertical displacements. The modal moment of inertia $\overline{\mathfrak{I}}_{3}$ is associated to the drilling rotations.

\section{Conclusions}

Nowadays Cosserat Finite Elements find numerous applications in mechanics and engineering. However, the details of the FE formulation are partially exposed in literature or not presented at all, and its performance is seldom investigated. Therefore, it is hard to assess the chosen formulation and to extend its applicability to other problems.

In this paper, a Cosserat dynamic Finite Element formulation for elastic plates has been presented and investigated in a detailed manner. In particular, a quadrilateral element with quadratic interpolation functions for both the displacement and the rotational fields has been proposed and validated through adequate patch tests and benchmarks. It has been demonstrated how, due to the non-symmetry of the stress tensor and the drilling rotation that is inherent to 
the micropolar continuum, this element has advantageous computational properties when compared to classical formulations. Local matrix deficiencies are not detected in the case of reduced integration, without the use of hourglass control techniques. Cosserat continuum introduces in a physical way the stiffness associated to the drilling rotations, which prevents the hourglass modes to occur and makes the presented formulation suitable for the assemblage of shell structures. The performance of the element in representing the bending behaviour in the limit of thin geometries has been also studied, showing a quite good performance against the shear locking phenomenon. The element has been then validated under dynamic conditions. Wave dispersion has been studied with respect to a Cosserat onedimensional configuration. The comparison between the results of the numerical model against the theoretical solution has demonstrated the precision of the formulation in modelling in-plane and out-of-plane modal shapes with high frequencies.

The presented Cosserat Finite Element formulation is very general and allows various constitutive laws for materials with micro-structure to be implemented. In order to highlight the practical interest of the formulation, an application has been given, in which a masonry panel made of rigid building blocks with deformable interfaces has been modeled through the validated finite element. The in- and out-of-plane modal response of masonry has been considered, by focusing on the shearing, flexural and torsional oscillation modes of the panel. The role of the microstructure has been also studied, by modelling panels with various aspect ratios and slenderness. The results from the homogenized Cosserat Finite Element analyses have been juxtaposed to those obtained by the use of an analogue Discrete Elements model, and a good agreement has been found. The role of the drilling rotations has been then assessed, showing their significant participation to the inertial response of the masonry panels against in-plane seismic excitations.

\section{Appendix 1}

Considering a Cosserat material without specific centrosymmetric properties and always supposing uncoupled inplane and out-of-plane behavior, Eq.(9) reads:

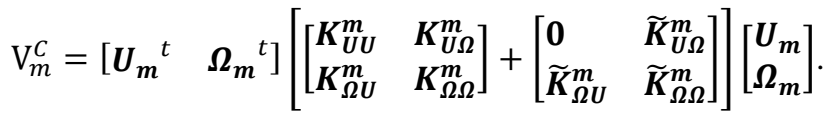

The additional terms are indicated with $[\widetilde{\cdot}]$ and are:

$$
\begin{aligned}
& \widetilde{\boldsymbol{K}}_{U \Omega}^{\boldsymbol{m}}=\int_{\Omega}\left[\boldsymbol{B}_{\boldsymbol{m 1}[2 N \times 4]}^{t} \boldsymbol{G}_{[4 \times 2]} \boldsymbol{B}_{\boldsymbol{m} 4[2 \times M]}\right] \mathrm{d} A
\end{aligned}
$$

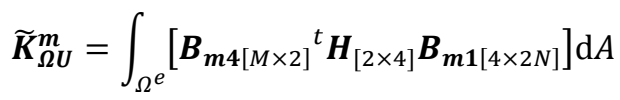

$$
\begin{aligned}
& \widetilde{\boldsymbol{K}}_{\boldsymbol{\Omega} \boldsymbol{\Omega}}^{\boldsymbol{m}}=h \int_{\Omega}{ }\left[\boldsymbol{B}_{\boldsymbol{m} 4[M \times 2]}{ }^{t} \boldsymbol{H}_{[2 \times 4]} \boldsymbol{B}_{\boldsymbol{m} 2[4 \times M]}+\boldsymbol{B}_{\boldsymbol{m} 2[M \times 4]}{ }^{t} \boldsymbol{G}_{[4 \times 2]} \boldsymbol{B}_{\boldsymbol{m} \mathbf{4}[2 \times M]}\right] \mathrm{d} A .
\end{aligned}
$$

\section{Appendix 2}

Let us consider the elastic deformation energy associated to the present Cosserat plate model due to the in-plane deformations:

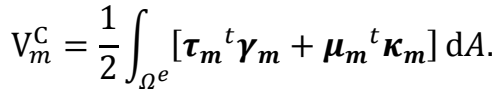

Taking into account the constitutive law (19), we obtain:

$$
\mathrm{V}_{m}^{\mathrm{C}}=\frac{1}{2} \int_{\Omega}\left[\boldsymbol{\gamma}_{\boldsymbol{m}}{ }^{t} \boldsymbol{A} \boldsymbol{\gamma}_{\boldsymbol{m}}+\boldsymbol{\kappa}_{\boldsymbol{m}}{ }^{t} \boldsymbol{D}_{\boldsymbol{m}} \boldsymbol{\kappa}_{\boldsymbol{m}}\right] \mathrm{d} A
$$

The derivation of the skew-symmetric part from the element formulation is made possible by separating every term of the strain vector into its symmetric and skew-symmetric part and by reordering the resulting components in a new vectorial form:

$$
\widetilde{\gamma}_{\boldsymbol{m}}=\left[\begin{array}{llll}
\gamma_{11} & \gamma_{22} & \gamma_{(12)} & \gamma_{[12]}
\end{array}\right]^{t}
$$




$$
\widetilde{\boldsymbol{\kappa}}_{\boldsymbol{m}}=\left[\begin{array}{ll}
\kappa_{31} & \kappa_{32}
\end{array}\right]^{t}=\boldsymbol{\kappa}_{\boldsymbol{m}} .
$$

The stress components can be also separated into their symmetric and skew-symmetric parts:

$$
\begin{aligned}
\tilde{\boldsymbol{\tau}}_{\boldsymbol{m}} & =\left[\begin{array}{llll}
\tau_{11} & \tau_{22} & \tau_{(12)} & \tau_{[12]}
\end{array}\right]^{t} \\
\widetilde{\boldsymbol{\mu}}_{\boldsymbol{m}} & =\left[\begin{array}{ll}
\mu_{31} & \mu_{32}
\end{array}\right]^{t}=\boldsymbol{\mu}_{\boldsymbol{m}} .
\end{aligned}
$$

A new constitutive law between (71) and (72) can be expressed through the basic linear relation

$$
\widetilde{A}=\frac{\partial^{2}}{\partial \widetilde{\gamma}_{m} \partial \widetilde{\gamma}_{m}}\left[\widetilde{\mathrm{V}}_{m}^{\mathrm{C}}\right]
$$

where $\widetilde{\mathrm{V}}_{m}^{\mathrm{C}}$ takes the form:

$$
\widetilde{\mathrm{V}}_{m}^{\mathrm{C}}=\frac{1}{2} \int_{\Omega^{e}}\left[\widetilde{\gamma}_{m}{ }^{t} \widetilde{\boldsymbol{A}} \widetilde{\gamma}_{m}+\boldsymbol{\kappa}_{m}{ }^{t} \boldsymbol{D}_{\boldsymbol{m}} \boldsymbol{\kappa}_{\boldsymbol{m}}\right] \mathrm{d} A .
$$

Thus, starting from matrix

$$
\boldsymbol{A}=\left[\begin{array}{llll}
A^{1111} & A^{1122} & & \\
A^{2211} & A^{2222} & & \\
& & A^{1212} & A^{1221} \\
& & A^{2112} & A^{2121}
\end{array}\right],
$$

we obtain:

$$
\widetilde{\boldsymbol{A}}=\left[\begin{array}{llll}
A^{1111} & A^{1122} & & \\
A^{2211} & A^{2222} & & \\
& & A^{(12)(12)} & A^{(12)[12]} \\
& & A^{[12](12)} & A^{[12][12]}
\end{array}\right]
$$

with

$$
\begin{aligned}
& A^{(12)(12)}=\left[A^{1212}+A^{1221}+A^{2121}+A^{2112}\right] / 2 \\
& A^{(12)[12]}=\left[A^{1212}-A^{1221}-A^{2121}+A^{2112}\right] / 2 \\
& A^{[12](12)}=\left[A^{1212}+A^{1221}-A^{2121}-A^{2112}\right] / 2 \\
& A^{[12][12]}=\left[A^{1212}-A^{1221}+A^{2121}-A^{2112}\right] / 2 .
\end{aligned}
$$

Cauchy continuum can be retrieved by annihilating the skew-symmetric terms contained in Eq.(78)-(80), which automatically guarantees the self-satisfaction of the balance equations, without the necessity of any couple stress addition. In the case that $\boldsymbol{A}$ is given by the first sub-matrix of Eq.(47), we have:

$$
A^{[12][12]}=2 G_{c}=2 G \alpha=2 A^{(12)(12)} \alpha \quad A^{(12)[12]}=A^{[12](12)}=0 .
$$

Therefore the term $\alpha$ controls in magnitude the skew-symmetric part of the shear stress within the constitutive law.

\section{Appendix 3}

Given $\boldsymbol{A}$ by Eq.(75) and $\boldsymbol{B}_{\boldsymbol{m} 2}$ by Eq.(27), the first term of the stiffness matrix in Eq.(31) reads:

$$
\boldsymbol{K}_{\Omega \Omega}^{m}{ }^{(1)}=\int_{\Omega^{e}}\left[\begin{array}{rrr}
0 & & 0 \\
0 & & 0 \\
\Phi_{1} & & \Phi_{N} \\
-\Phi_{1} & \ldots & -\Phi_{N}
\end{array}\right]^{t}\left[\begin{array}{llll}
A^{1111} & A^{1122} & & \\
A^{2211} & A^{2222} & & \\
& & A^{1212} & A^{1221} \\
& & A^{2112} & A^{2121}
\end{array}\right]\left[\begin{array}{rrr}
0 & 0 \\
0 & & 0 \\
\Phi_{1} & & \Phi_{N} \\
-\Phi_{1} & \ldots & -\Phi_{N}
\end{array}\right] \mathrm{d} A
$$

Recalling the definitions (18) and (80), the above matrix is:

$$
\boldsymbol{K}_{\boldsymbol{\Omega} \Omega}^{\boldsymbol{m}}{ }^{(1)}=\int_{\Omega^{e}} 2 A^{[12][12]} \boldsymbol{\Phi}_{\boldsymbol{m}} \otimes \boldsymbol{\Phi}_{\boldsymbol{m}} d A=\int_{\Omega^{e}} 2 A^{[12][12]} \boldsymbol{\Phi}_{\boldsymbol{m}}{ }^{t} \boldsymbol{\Phi}_{\boldsymbol{m}} \mathrm{d} A,
$$


where the outer product $(\cdot \otimes \cdot)$ has been expressed as a scalar product, with $\boldsymbol{\Phi}_{\boldsymbol{m}}$ from Eq.(24). We note that only the matrix $\boldsymbol{K}_{\Omega \Omega}^{\boldsymbol{m}}{ }^{(1)}$ contains the elastic modulus $A^{[12][12]}$ associated to the Cosserat's term responsible for the hourglass control, i.e. $\gamma_{[12]}$. Writing the elastic potential associated to that matrix as:

$$
\mathrm{V}_{\Omega \Omega}^{C m}{ }^{(1)}=\frac{1}{2} \boldsymbol{\Omega}_{m}{ }^{t} \boldsymbol{K}_{\Omega \Omega}^{m}{ }^{(1)} \boldsymbol{\Omega}_{\boldsymbol{m}},
$$

and by introducing Eq.(83), we have:

$$
\mathrm{V}_{\Omega \Omega}^{C m(1)}=\frac{1}{2} \int_{\Omega}\left[\boldsymbol{\Phi}_{\boldsymbol{m}} \boldsymbol{\Omega}_{\boldsymbol{m}}\right]^{t} 2 A^{[12][12]}\left[\boldsymbol{\Phi}_{\boldsymbol{m}} \boldsymbol{\Omega}_{\boldsymbol{m}}\right] \mathrm{d} A .
$$

In the above we recognise the second expression of Eq.(22). It results in:

$$
\mathrm{V}_{\Omega \Omega}^{C m(1)}=\frac{1}{2} \int_{\Omega} 2 A^{[12][12]} \omega_{3}^{2} \mathrm{~d} A .
$$

So $\boldsymbol{K}_{\Omega \Omega}^{\boldsymbol{m}}{ }^{(1)}$ depends on the drilling rotations $\omega_{3}$ and if $A^{[12][12]}=2 G \alpha$ (Eq. (81)), then $\alpha$ gives a weight to the drilling rotations in the assemblage of the element membrane stiffness matrix.

The above potential is similar to that generally proposed by [44] for the construction of shell structures by assemblage of flat FE. This latter can be expressed as:

$$
\mathrm{V}_{\Omega \Omega}^{P K}(1)=\beta \gamma \int_{\Omega}\left[\widehat{\omega}_{30}-\omega_{3}\right]^{2} \mathrm{~d} A \approx \beta \gamma \int_{\Omega^{e}} \gamma_{[12]}^{2} \mathrm{~d} A,
$$

where $\widehat{\omega}_{30}$ the average infinitesimal rotation (Eq.(4)) of the element, $\beta$ depends on the material and $\gamma$ is an arbitral parameter thought which one makes the stiffness matrix non-singular, against possible membrane actions.

\section{References}

[1] I. Vardoulakis and J. Sulem, Bifurcation analysis in geomechanics. Glasgow: Blackie Academic and Professional, 1995, p. 484

[2] E. Pasternak and H.-B. Mühlhaus, "Generalised homogenisation procedures for granular materials," J. Eng. Math., vol. 52, no. 1, pp. 199-229, Jul. 2005.

[3] I. Vardoulakis, "Shear-banding and liquefaction in granular materials on the basis of a Cosserat continuum theory," Ingenieur-Archiv, vol. 59, no. 2, pp. 106-113, 1989.

[4] J. Sulem, M. Veveakis, and I. Stefanou, "Failure in shear bands for granular materials: thermo-hydrochemo-mechanical effects," Géotechnique Lett., vol. 3, no. April-June, pp. 31-36, May 2013.

[5] P. de Buhan, J. Fréard, D. Garnier, and S. Maghous, "Failure Properties of Fractured Rock Masses as Anisotropic Homogenized Media,” J. Eng. Mech., vol. 128, no. 8, pp. 869-875, Aug. 2002.

[6] J. Sulem, I. Stefanou, and E. Veveakis, "Stability analysis of undrained adiabatic shearing of a rock layer with Cosserat microstructure," Granul. Matter, vol. 13, no. 3, pp. 261-268, Feb. 2011.

[7] J. Sulem and H.-B. Mühlhaus, "A continuum model for periodic two-dimensional block structures," Mech. Cohesive-frictional Mater., vol. 2, no. 1, pp. 31-46, Jan. 1997.

[8] I. Stefanou, J. Sulem, and I. Vardoulakis, "Three-dimensional Cosserat homogenization of masonry structures: elasticity,” Acta Geotech., vol. 3, no. 1, pp. 71-83, Feb. 2008.

[9] A. Pau and P. Trovalusci, "Block masonry as equivalent micropolar continua: the role of relative rotations," Acta Mech., vol. 223, no. 7, pp. 1455-1471, May 2012.

[10] S. Forest and K. Sab, "Cosserat overall modeling of heterogeneous materials," Mech. Res. Commun., vol. 25, no. 4, pp. 449-454, Jul. 1998. 
[11] S. Forest, F. Pradel, and K. Sab, “Asymptotic analysis of heterogeneous Cosserat media,” Int. J. Solids Struct., vol. 38, no. 26-27, pp. 4585-4608, Jun. 2001.

[12] M. Dehghani and S. A. A. Moosavian, "Static modeling of continuum robots by circular elements," in 2013 21 st Iranian Conference on Electrical Engineering (ICEE), 2013, pp. 1-6.

[13] G. Robinson and J. B. C. Davies, "Continuum robots - a state of the art," Proc. - IEEE Int. Conf. Robot. Autom., vol. 4, pp. 2849-2854, 1999.

[14] L. Li and S. Xie, "Finite element method for linear micropolar elasticity and numerical study of some scale effects phenomena in MEMS," Int. J. Mech. Sci., vol. 46, no. 11, pp. 1571-1587, Nov. 2004.

[15] C. Tekoglu and P. Onck, "Size effects in two-dimensional Voronoi foams: A comparison between generalized continua and discrete models," J. Mech. Phys. Solids, vol. 56, no. 12, pp. 3541-3564, Dec. 2008.

[16] A. C. Eringen, Microcontinuum field theories. I: Foundations and solids. New York: Springer, 1999 , p. 325.

[17] A. C. Eringen, Microcontinuum field theories. II: Fluent media. New York: Springer, 2001, p. 341.

[18] H. C. Park and R. S. Lakes, "Cosserat micromechanics of human bone: Strain redistribution by a hydration sensitive constituent,” J. Biomech., vol. 19, no. 5, pp. 385-397, 1986.

[19] J. Fatemi, F. Van Keulen, and P. R. Onck, "Generalized continuum theories: Application to stress analysis in bone," in Meccanica, 2002, vol. 37, no. 4-5, pp. 385-396.

[20] I. Goda, M. Assidi, and J. F. Ganghoffer, "A 3D elastic micropolar model of vertebral trabecular bone from lattice homogenization of the bone microstructure.," Biomech. Model. Mechanobiol., vol. 13, no. 1, pp. 5383, Jan. 2014.

[21] D. Sanchez-Molina, J. Velazquez-Ameijide, C. Arregui-Dalmases, D. Rodríguez, V. Quintana, M. Shafieian, and J. R. Crandall, "A microcontinuum model for mechanical properties of esophageal tissue: experimental methodology and constitutive analysis.," Ann. Biomed. Eng., vol. 42, no. 1, pp. 62-72, Jan. 2014.

[22] K. Sack, S. Skatulla, and C. Sansour, "Research and Applications in Structural Engineering, Mechanics and Computation," in Proceedings of the 5th International Conference on Structural Engineering, Mechanics and Computation (SEMC), 2013, 2013, pp. 423-427.

[23] J. Altenbach, H. Altenbach, and V. a. Eremeyev, "On generalized Cosserat-type theories of plates and shells: a short review and bibliography," Arch. Appl. Mech., vol. 80, no. 1, pp. 73-92, Sep. 2009.

[24] R. de Borst and L. Sluys, "Localisation in a Cosserat continuum under static and dynamic loading conditions," Comput. Methods Appl. Mech. Eng., vol. 90, no. 1-3, pp. 805-827, Sep. 1991.

[25] R. de Borst and L. Sluys, "Fundamental issues in finite element analyses of localization of deformation," Eng. Comput., vol. 10, no. 2, pp. 99-121, 1993.

[26] R. DE BORST, "SIMULATION OF STRAIN LOCALIZATION: A REAPPRAISAL OF THE COSSERAT CONTINUUM," Eng. Comput., vol. 8, no. 4, pp. 317-332, 1991.

[27] A. Riahi, J. H. Curran, and H. Bidhendi, "Buckling analysis of 3D layered structures using a Cosserat continuum approach," Comput. Geotech., vol. 36, no. 7, pp. 1101-1112, Sep. 2009.

[28] A. Riahi and J. H. Curran, "Full 3D finite element Cosserat formulation with application in layered structures," Appl. Math. Model., vol. 33, no. 8, pp. 3450-3464, Aug. 2009.

[29] C. Dai, H. Muhlhaus, M. Duncan Fama, and J. Meek, "Finite element analysis of Cosserat Theory for layered rock masses," Comput. Geotech., vol. 15, no. 3, pp. 145-162, Jan. 1993. 
[30] M. Cerrolaza, J. Sulem, and A. Elbied, "A Cosserat non-linear finite element analysis software for blocky structures,” Adv. Eng. Softw., vol. 30, no. 1, pp. 69-83, 1999.

[31] D. Addessi, E. Sacco, and A. Paolone, "Cosserat model for periodic masonry deduced by nonlinear homogenization,” Eur. J. Mech. - A/Solids, vol. 29, no. 4, pp. 724-737, Jul. 2010.

[32] D. Addessi and E. Sacco, "A multi-scale enriched model for the analysis of masonry panels," Int. J. Solids Struct., vol. 49, no. 6, pp. 865-880, Mar. 2012.

[33] D. Addessi, "A 2D Cosserat finite element based on a damage-plastic model for brittle materials," Comput. Struct., vol. 135, pp. 20-31, Apr. 2014.

[34] P. Trovalusci and A. Pau, "Derivation of microstructured continua from lattice systems via principle of virtual works: the case of masonry-like materials as micropolar, second gradient and classical continua," Acta Mech., vol. 225, no. 1, pp. 157-177, Aug. 2014.

[35] J. Chróścielewski and W. Witkowski, “On some constitutive equations for micropolar plates,” ZAMM, vol. 90, no. 1, pp. 53-64, Jan. 2010.

[36] J. Chróścielewski and W. Witkowski, "FEM analysis of Cosserat plates and shells based on some constitutive relations," ZAMM - J. Appl. Math. Mech. / Zeitschrift für Angew. Math. und Mech., vol. 91, no. 5, pp. 400-412, May 2011.

[37] B. Nadler and M. B. Rubin, "Determination of hourglass coefficients in the theory of a Cosserat point for nonlinear elastic beams,” Int. J. Solids Struct., vol. 40, no. 22, pp. 6163-6188, Nov. 2003.

[38] M. Jabareen and M. B. Rubin, “A six node plane strain triangular Cosserat Point Element (CPE) for nonlinear elasticity,” Int. J. Eng. Sci., vol. 74, pp. 118-142, Jan. 2014.

[39] E. Mtanes and M. Jabareen, "A plane strain quadrilateral Cosserat point element (CPE) for nonlinear orthotropic elastic materials - An extension to initially distorted geometry and general orthotropic directions," Finite Elem. Anal. Des., vol. 87, pp. 10-21, Sep. 2014.

[40] A. Zervos, "Finite elements for elasticity with microstructure and gradient elasticity," Int. J. Numer. Methods Eng., vol. 73, no. 4, pp. 564-595, 2008.

[41] O. Zienkiewicz and R. Taylor, The finite element method for solid and structural mechanics, Sixth. Oxford: Elsevier Butterworth-Heinemann, 2005, p. 631.

[42] T. J. R. Hughes and F. Brezzi, “On drilling degrees of freedom,” Comput. Methods Appl. Mech. Eng., vol. 72, no. 1, pp. 105-121, Jan. 1989.

[43] C. Chinosi, M. Comodi, and G. Sacchi, "A new finite element with 'drilling' D.O.F.," Comput. Methods Appl. Mech. Eng., vol. 143, no. 1-2, pp. 1-11, Apr. 1997.

[44] E. Providas and M. a. Kattis, "An assessment of two fundamental flat triangular shell elements with drilling rotations," Comput. Struct., vol. 77, no. 2, pp. 129-139, Jun. 2000.

[45] S. Kugler, P. a. Fotiu, and J. Murin, "A highly efficient membrane finite element with drilling degrees of freedom,” Acta Mech., vol. 213, no. 3-4, pp. 323-348, Feb. 2010.

[46] H. T. Y. Yang, S. Saigal, A. Masud, and R. K. Kapania, “A survey of recent shell finite elements,” Int. J. Numer. Methods Eng., vol. 47, no. 1-3, pp. 101-127, Jan. 2000.

[47] P. Germain, "The method of virtual power in continuum mechanics. Part 2: Microstructure," SIAM J. Appl. Math., vol. 25, no. 3, pp. 556-575, 1973.

[48] H. Schaefer, "Analysis der Motorfelder im Cosserat-Kontinuum,” ZAMM - J. Appl. Math. Mech. /Zeitschrift für Angew. Math. und Mech., vol. 47, pp. 319-328, 1967. 
[49] I. Vardoulakis, "Lecture notes on Cosserat continuum mechanics with application to the mechanics of granular media," 3 National Meeting on "Generalized Continuum Theories and Applications”, Thessaloniki, 2009. [Online]. Available: http://geolab.mechan.ntua.gr/teaching/lectnotes/CCM2009.pdf. [Accessed: 09Nov-2013].

[50] A. C. Eringen, “Theory of micropolar plates," ZAMP - Zeitschrift für Angew. Math. und Phys., vol. 18, no. 1, pp. 12-30, Jan. 1967.

[51] G. A. Gevorkyan, "The basic equations of flexible plates for a medium of cosserat," Sov. Appl. Mech., vol. 3, no. 11, pp. 41-45, Nov. 1967.

[52] S. Ambartsumian, "The theory of transverse bending of plates with asymmetric elasticity," Mech. Compos. Mater., vol. 32, no. 1, pp. 30-38, 1996.

[53] H. Altenbach and V. A. Eremeyev, "On the linear theory of micropolar plates," ZAMM - J. Appl. Math. Mech. / Zeitschrift für Angew. Math. und Mech., vol. 89, no. 4, pp. 242-256, Apr. 2009.

[54] V. A. Eremeyev and W. Pietraszkiewicz, "Local Symmetry Group in the General Theory of Elastic Shells," J. Elast., vol. 85, no. 2, pp. 125-152, Aug. 2006.

[55] V. a. Eremeyev, L. P. Lebedev, and H. Altenbach, Foundations of Micropolar Mechanics. Berlin, Heidelberg: Springer Berlin Heidelberg, 2013, p. 142.

[56] E. Providas and M. a. Kattis, "Finite element method in plane Cosserat elasticity," Comput. Struct., vol. 80, no. 27-30, pp. 2059-2069, Nov. 2002.

[57] I. Stefanou, J. Sulem, and I. Vardoulakis, "Homogenization of interlocking masonry structures using a generalized differential expansion technique,” Int. J. Solids Struct., vol. 47, no. 11-12, pp. 1522-1536, Jun. 2010 .

[58] Simulia, “Abaqus 6.10.” 2010.

[59] H. Zhang, H. Wang, and G. Liu, "Quadrilateral isoparametric finite elements for plane elastic Cosserat bodies," Acta Mech. Sin., vol. 21, no. 4, pp. 388-394, Jun. 2005.

[60] P. Unterreiner, “Contribution à l'étude et à la modélisation numérique des sols cloués: application au calcul en déformation des ouvrages de soutènement," Ecole des Ponts et Chaussées, 1994.

[61] T. Hughes, The finite element method: linear static and dynamic finite element analysis. New Jersey: Prentice-Hall, 1987.

[62] F. François and J. Jaroslav, Analyse des structures et milieux continus - Volume 6: Méthode des éléments finis. Lausanne: Presses polytechniques et universitaires romandes, 2009.

[63] K. Bathe, Finite element procedures. New Jersey: Prentice Hall, 1996.

[64] R. L. Taylor and S. Govindjee, "Solution of clamped rectangular plate problems," Commun. Numer. Methods Eng., vol. 20, no. 10, pp. 757-765, Jul. 2004.

[65] Itasca Consulting Group, “3DEC 5.0.” Minneapolis, 2013.

[66] P. Cundall, "Formulation of a three-dimensional distinct element model-Part I. A scheme to detect and represent contacts in a system composed of many polyhedral blocks," Int. J. Rock Mech. Min. Sci. Geomech. Abstr., vol. 25, no. 3, pp. 107-116, Jun. 1988.

[67] J. V. Lemos, "Discrete Element Modeling of Masonry Structures," Int. J. Archit. Herit., vol. 1, no. 2, pp. 190-213, May 2007. 


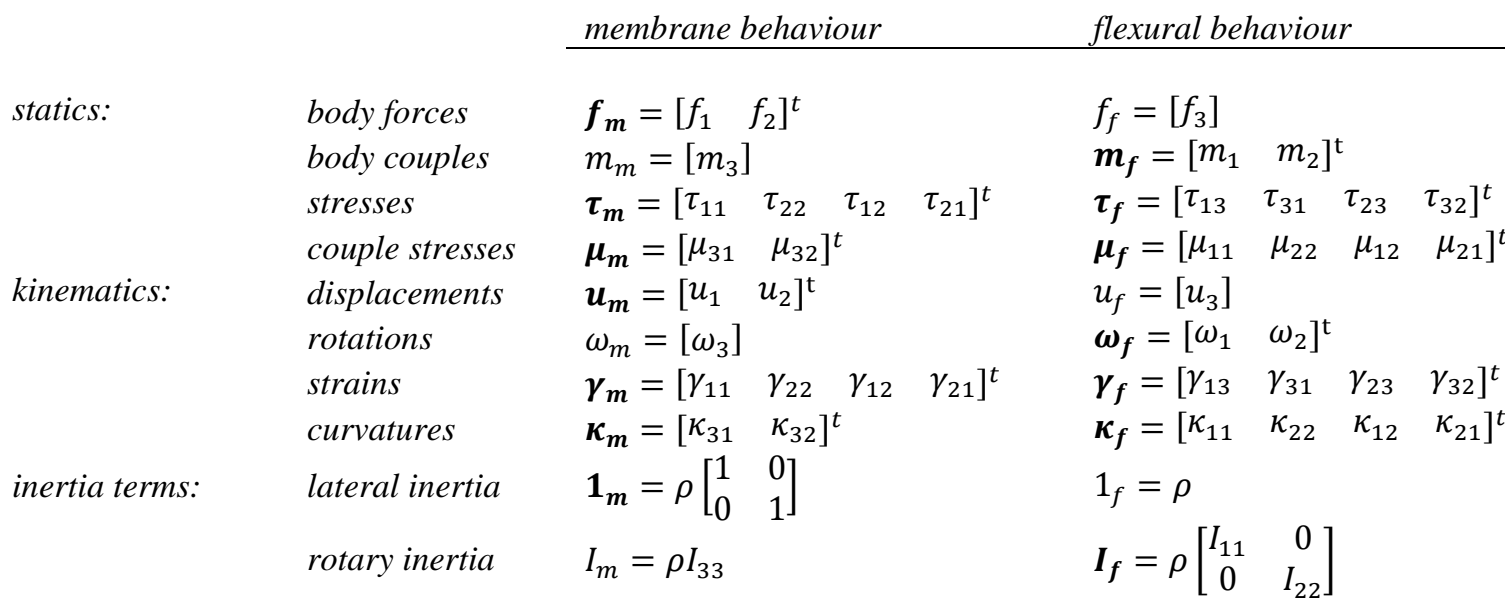

Table 1 Field variables of the Cosserat plate-type model in matrix notation. Membrane and flexural behaviour. The components of the inertia tensor are represented in the principal basis.

\begin{tabular}{|c|c|c|c|c|}
\hline & \multirow[b]{2}{*}{ element formulation } & \multirow[b]{2}{*}{ K } & \multicolumn{2}{|c|}{ number of integration points } \\
\hline & & & $2 \times 2$ & $3 \times 3$ \\
\hline (A) & Cosserat plate & Eq.(29)-(31), (34)-(37) & 0 & 0 \\
\hline (B) & Cosserat membrane & Eq. $(29)-(31)$ & 0 & 0 \\
\hline (C) & Cauchy membrane & Eq.(29) & 1 & 0 \\
\hline
\end{tabular}

Table 2 Computed number of zero-energy modes other than those associated with rigid body modes.

Test 1

\begin{tabular}{llll}
\hline$u_{1}=10^{-3}(x+0.5 y)$ & $u_{2}=10^{-3}(x+y)$ & $\omega_{3}=0.25 \times 10^{-3}$ & $f_{1}=f_{2}=0 m_{3}=0$ \\
$\tau_{11}=\tau_{22}=4$ & $\tau_{12}=\tau_{21}=1.5$ & $\mu_{31}=\mu_{32}=0$ &
\end{tabular}

Test 2

\begin{tabular}{llll}
\hline$u_{1}=10^{-3}(x+0.5 y)$ & $u_{2}=10^{-3}(x+y)$ & $\omega_{3}=10^{-3}\left(0.25+(4 \alpha)^{-1}\right)$ & $f_{1}=f_{2}=0 m_{3}=1$ \\
$\tau_{11}=\tau_{22}=4$ & $\tau_{12}=2 \quad \tau_{21}=1$ & $\mu_{31}=\mu_{32}=0$ & \\
& & & \\
Test 3 & & & \\
\hline$u_{1}=10^{-3}(x+0.5 y)$ & $u_{2}=10^{-3}(x+y)$ & $\omega_{3}=10^{-3}\left[0.25+(2 \alpha)^{-1}(x-y)\right]$ & $f_{1}=f_{2}=1 m_{3}=2(x-y)$ \\
$\tau_{11}=\tau_{22}=4$ & $\tau_{12}=1.5+(x-y)$ & $\mu_{31}=-\mu_{32}=4$. & \\
& $\tau_{21}=1.5-(x-y)$ & &
\end{tabular}

Table 3 Patch test for Cosserat plane elements: boundary conditions and expected solutions.

\begin{tabular}{lllllll} 
& $\tau_{11}$ & $\tau_{22}$ & $\tau_{12}$ & $\tau_{21}$ & $\mu_{31}$ & $\mu_{32}$ \\
\hline Test1 & 4.000 & 4.000 & 1.500 & 1.500 & 0.000 & 0.000 \\
& {$[4.000]$} & {$[4.000]$} & {$[1.500]$} & {$[1.500]$} & {$[0.000]$} & {$[0.000]$} \\
Test2 & 4.000 & 4.000 & 1.000 & 2.000 & 0.010 & 0.022 \\
& {$[4.000]$} & {$[4.000]$} & {$[1.000]$} & {$[2.000]$} & {$[0.000]$} & {$[0.000]$} \\
Test3 & 3.976 & 3.970 & 1.521 & 1.447 & 3.997 & -4.001 \\
& {$[4.000]$} & {$[4.000]$} & {$[1.537]$} & {$[1.462]$} & {$[4.000]$} & {$[-4.000]$}
\end{tabular}

Table 4 Results of patch tests at point $P 1$. Exact results are reported in parenthesis. 